\title{
TM4SF5-mediated liver malignancy involves NK cell exhaustion-like phenotypes
}

\author{
Hyunseung Sun ${ }^{1,2} \cdot$ Eunmi Kim $^{1,2} \cdot$ Jihye Ryu ${ }^{1,2} \cdot$ Hyejin Lee $^{1} \cdot$ Eun-Ae Shin ${ }^{1} \cdot$ Minhyeong Lee $^{1} \cdot$ Haesong Lee $^{1}$. \\ Jeong-Hoon Lee ${ }^{3} \cdot$ Jung-Hwan Yoon ${ }^{3} \cdot$ Dae-Geun Song ${ }^{4} \cdot$ Semi Kim $^{5} \cdot$ Jung Weon Lee ${ }^{1,2}$ (i)
}

Received: 13 July 2021 / Revised: 8 November 2021 / Accepted: 18 November 2021 / Published online: 18 December 2021

(c) The Author(s) 2021

\begin{abstract}
Aberrant extracellular matrix and immune cell alterations within the tumor microenvironment promote the pathological progression of liver carcinogenesis. Although transmembrane $4 \mathrm{~L}$ six family member 5 (TM4SF5) is involved in liver fibrosis and cancer, its mechanism avoiding immune surveillance during carcinogenesis remains unknown. We investigated how TM4SF5-mediated signaling caused immune evasion using in vitro primary cells and in vivo liver tissues from genetic or chemically induced mouse models. TM4SF5-transgenic and diethylnitrosamine (DEN)-induced liver cancer mouse models exhibited fibrotic and cancerous livers, respectively, with enhanced TM4SF5, pY ${ }^{705}$ STAT3, collagen I, and laminin $\gamma 2$ levels. These TM4SF5-mediated effects were abolished by TM4SF5 inhibitor, 4'-( $p$-toluenesulfonylamido)-4-hydroxychalcone (TSAHC). TM4SF5-dependent tumorigenesis involved natural killer (NK) cell exhaustion-like phenotypes including the reduction of NK cell number or function, which were blocked with TSAHC treatment. TM4SF5 expression in cancer cells downregulated stimulatory ligands and receptors for NK cell cytotoxicity, including SLAMF6, SLAMF7, MICA/B, and others. TM4SF5 suppression or inhibition reduced STAT3 signaling activity and recovered the receptor levels and NK cell surveillance, leading to reduced fibrotic and cancerous phenotypes, and longer survival. Altogether, these findings suggest that TM4SF5-mediated STAT3 activity for extracellular matrix modulation is involved in the progression of liver disease to HCC and that TM4SF5 appears to suppress NK cells during liver carcinogenesis.
\end{abstract}

Keywords Immune checkpoint · NK cell immune therapy · Liver cancer · Signal transduction $\cdot$ L6 family member

\section{Introduction}

Hyunseung Sun and Eunmi Kim contributed equally.

Jung Weon Lee

jwl@snu.ac.kr

1 Department of Pharmacy, College of Pharmacy, Seoul National University, Seoul 08826, Republic of Korea

2 Research Institute of Pharmaceutical Sciences, College of Pharmacy, Seoul National University, Seoul 08826, Republic of Korea

3 Department of Internal Medicine and Liver Research Institute, Seoul National University College of Medicine, Seoul 03080, Republic of Korea

4 Natural Product Informatics Research Center, Korea Institute of Science and Technology (KIST), Gangneung-si, Gangwon-do 25451, Republic of Korea

5 Immunotherapy Convergence Research Center, Korea Research Institute of Bioscience and Biotechnology, Daejon 34141, Republic of Korea
Chronic liver injury progressively causes liver diseases, including nonalcoholic fatty liver disease (NAFLD), via excessive production of the extracellular matrix (ECM), which drives fibrosis and cirrhosis and ultimately results in hepatocellular carcinoma (HCC) [1]. Specifically, the excessive ECM deposition during fibrosis and cirrhosis activates abnormal cell proliferation signaling for $\mathrm{HCC}$ development [2].

Nonalcoholic injury-mediated liver inflammation results in the production of diverse cytokines, which can facilitate ECM production for preneoplastic progression and eventual carcinogenesis. In addition to the influence of the inflammatory environment, immune cells in the microenvironment are critically associated with the carcinogenic processes [3]. After their transformation from normal to malignant cells, cancer cells present tumor antigens to $\mathrm{T}$ cells, and antigenpresenting cells (APS, including dendritic cells, B cells, and 
macrophages) play a crucial role as nexus, performing crosspresentation of tumor-derived antigens. The identification of cancer-associated antigens by APCs then leads to T-cell priming and tumor infiltration, which eventually leads to T-cell-mediated recognition and killing of the tumor cells [3]. During this process, immune checkpoints, such as cytotoxic T lymphocytic protein 4 (CTLA-4) and programmed cell death protein 1 (PD-1), on immune cell surfaces have been actively targeted to boost the immune system and for anti-tumor immunotherapies [4]. Recently, the anti-cancer efficacy of antibodies against PD-1/programmed cell death ligand 1 (PD-L1) via T-cell checkpoint blockade in obese cancer patients [5] and non-small cell lung cancer [6]. Despite significant immune surveillance in the liver, HCC can still occur as a result of chronic liver infection or inflammation. Natural killer (NK) cells make up approximately $50 \%$ of the liver lymphocyte population and kill tumors via the release of pro-inflammatory cytokine interferon- $\gamma$ (IFN$\gamma$ ) [7] and cytotoxic granules, such as perforin and granzyme [8], as well as via death receptor signaling (TNF-related apoptosis-inducing ligand [TRAIL] and Fas Ligand [FasL]) [9]. Unlike cytotoxic $\mathrm{CD} 8^{+} \mathrm{T}$-cells, $\mathrm{NK}$ cells are activated through a cascade of various activation/inactivation receptors on their cell surfaces, rather than by antigen presentation [10]. NK cell-mediated cytotoxicity relies on "missing-self" and "induced-self" recognition to identify target cancer cells by maintaining a precise balance between co-stimulatory and co-inhibitory signals via functional receptors. These interacting signals eventually lead to the activation and functional status of NK cells [11]. Because the liver provides a robustly immunosuppressive microenvironment compared to other organs and contains a large population of NK cells that express immune checkpoints, it is the least responsive organ to current checkpoint immunotherapies in patients with liver cancer and metastases [12]. The high frequency of liver-resident NK cells in the healthy liver also suggests an essential role of NK cells in targeting and preventing liver metastasis. Although we currently have a good understanding of the complex and often redundant activation/inactivation pathways of NK cells [13], HCC-specific checkpoints in hepatocytes and NK cells warrant further investigation.

Transmembrane $4 \mathrm{~L}$ six family member 5 (TM4SF5, L6H also as a member of L6 four transmembrane superfamily [14]) is an $N$-glycosylated membrane protein with four transmembrane domains [15]. It is induced by transforming growth factor $\beta$ (TGF $\beta 1$ ) signaling in a carbon tetrachloride $\left(\mathrm{CCl}_{4}\right)$-induced liver fibrosis animal model [16] and is also highly expressed in clinical human liver cancer tissues [17], in addition to increased expression in colon, prostate, gastric, pancreatic, and esophageal cancers $[18,19]$. Macrophages, hepatic stellate cells, and endothelial cells in liver also show to express TM4SF5, with being increased upon inflammatory environment (data not shown).
TM4SF5 activates non-receptor tyrosine kinase c-SRC via direct physical association [20], which leads to signal transducer and activator of transcription 3 (STAT3) activation [21]. Although TM4SF5 is involved in animal liver fibrosis and xenograft growth, the mechanism by which TM4SF5 is influenced by the immune system, particularly via NK cells, during hepatic carcinogenesis remains unknown.

In this study, the roles of TM4SF5 in the development of precancerous and cancerous phenotypes in genetically engineered or chemically treated animals were explored in regard to TM4SF5-dependent signaling and crosstalk between cancer cells and immune cells. We found that TM4SF5-mediated STAT3 activity caused excessive ECM deposition during hepatic carcinogenesis, which was blocked by TM4SF5 inhibition, using an anti-TM4SF5 small compound. TM4SF5 inhibition or suppression downregulated co-stimulatory activation ligands in hepatocytes, which caused decreased cognate receptor expression on NK cells and eventually led to immune surveillance of TM4SF5-positive tumors. Thus, TM4SF5 is a potential target for NK cell-related immunotherapy against HCC.

\section{Materials and methods}

\section{Cells}

Human hepatocarcinoma cell lines that lacked TM4SF5 expression (SNU449 and empty-vector control SNU449C $\mathrm{C}_{\mathrm{p}}$ cells) or that expressed wild-type (WT) TM4SF5 [(exogenous expression) $\mathrm{SNU}_{449 \mathrm{~T}_{7}}$, (endogenous expression) HepG2, Huh7, and HepG2 cells] were used for in vitro experiments and were previously described [17]. Cells were purchased from either the Korean Cell Bank (Seoul, Korea) or American Type Culture Collection (ATCC, Manassas, VA, USA). Hepatocytes were cultured in Roswell Park Memorial Institute (RPMI) 1640 Medium or Dulbecco's Modified Eagle Medium (DMEM) (Cytiva, Marlborough, MA, USA) supplemented with $10 \%$ fetal bovine serum (FBS) and 1\% penicillin/streptomycin (GenDEPOT Inc., Barker, TX, USA) at $37{ }^{\circ} \mathrm{C}$ in $5 \% \mathrm{CO}_{2}$. Cells were stably infected with empty pLJM1-EGFP (Addgene) or TM4SF5encoding pLJM1-EGFP lentiviral vectors. Human NK92 cells were cultured in $\alpha$-Minimum Essential Medium (MEM) (Invitrogen, Grand Island, NY, USA) containing $200 \mathrm{U} / \mathrm{mL}$ recombinant human interleukin (IL)-2 (PeproTech, Rocky Hill, NJ, USA), $12.5 \%$ FBS, and $12.5 \%$ fetal horse serum (GenDEPOT Inc.). Stable cells were cultured in RPMI 1640 (WelGene) containing 10\% FBS, geneticin (G418; $250 \mu \mathrm{g} / \mathrm{mL}$ ), and antibiotics (Invitrogen, Grand Island, NY, USA). Cells were passaged every 3-4 days at ratios specified by the suppliers. Cells were monitored for mycoplasma contamination. 


\section{Transfection and infection}

The shRNA or cDNA expression plasmids were transfected for $48 \mathrm{~h}$ using Lipofectamine RNAiMAX or Lipofectamine 3000 (Thermo Fisher Scientific, Waltham, MA, USA), respectively, according to the manufacturer's protocols. Alternatively, cells were infected with lentivirus for shRNA or cDNA expression for $24 \mathrm{~h}$. Lentiviruses encoding shRNA against TM4SF5 (5'-CCGGACCATGTGTACGGGAAA ATGTGCCTCGAGGCACATTTTCCCGTACACATGGTT TTTTG-3' for sequence \#2, 5'-CCGGCCATCTCAGCTTGC AAGTCCTCGAGGACTTGCAAGCTGAGATGGTTTTTG $-3^{\prime}$ for sequence \#4) were prepared using lentiviral vector pLKO.1 (Addgene). Infected cells were selected with puromycin $(2 \mu \mathrm{g} / \mathrm{mL}$, GenDEPOT).

\section{Mice}

Age-matched WT and Tm4sf5-knockout $\left(\operatorname{Tm} 4 s f 5^{-1-}\right)$ C57BL/6 male mice as well as WT and TM4SF5-transgenic $\left(\mathrm{Tg}^{\mathrm{TM} 4 \mathrm{SF} 5}\right) \mathrm{FVB} / \mathrm{N}$ male mice were used for in vivo experiments. $\mathrm{Tg}^{\mathrm{TM} 4 \mathrm{SF} 5} \mathrm{FVB} / \mathrm{N}$ mice with a CMV promoter on the Tg construct for expression throughout the entire mouse were generated by breeding WT FVB/N and $\mathrm{Tg}^{\mathrm{TM} 4 \mathrm{SF5}} \mathrm{C} 57 \mathrm{BL} / 6$ mice [22] for 10 generations. The FVB/N strain was selected because it is susceptible to spontaneous development of lung cancer, rather than cancer of any other organs, including the liver. Furthermore, the ratio of liver tumors is lower in $\mathrm{FVB} / \mathrm{N}$ mice than in other strains [23]. $T m 4 s f 5^{-/-}$C57BL/6 mice were generated via embryo injection and transfer to normal, healthy female C57BL/6 recipient mice (Macrogen, Seoul, Korea), as described previously [24]. Briefly, the exon 1 of C57BL/6N-Tac Tm4sf5 gene by Cas 9 proteins single guide RNAs $\left(50 \mu \mathrm{g}, 5^{\prime}\right.$-GAG GTTGCCGTCCGTCCAGGTGG-3' and 5'-GCTGAGGTT GCCGTCCGTCCAGG-3' was targeted, and its deletion was proved by genotyping using primers for mouse- $T m 4 s f 5$ (forward 5'-CCAAGCCTCCCACCTGTTA-3', reverse 5'-GCTCCAGCATTCTCACCATC-3' for $\mathrm{KO}_{\text {exon1 } 1}$ ). We further regularly backcrossed the KO mice with WT mice. F1 heterozygous littermates ( $\operatorname{Tm} 4 s f 5^{-/+}$) were bred to generate homozygous mice $\left(\mathrm{Tm} 4 \mathrm{sf5^{-- }}\right)$. The littermates of WT, $\mathrm{Tm} 4 \mathrm{sf5^{-/ }}$ C57BL/6, WT or $\mathrm{Tg}^{\mathrm{TM} 4 \mathrm{SF} 5} \mathrm{FVB} / \mathrm{N}$ mice (isolated from a variety of different $\mathrm{C} 57 \mathrm{BL} / 6$ or $\mathrm{FVB} / \mathrm{N}$ litters, respectively) were used in experiments after randomized assignment following genotyping.

\section{Chemically induced animal models}

Four-week-old mice (BALB/c) were purchased from Orient. Co. Ltd (Seungnam, Korea). The mice were housed in a specific pathogen-free room with controlled temperature and humidity. Five-week-old mice $(n \geq 5)$ were injected intraperitoneally with or without $\mathrm{CCl}_{4}$ (Sigma-Aldrich, St. Louis, MO, USA; $1 \mathrm{mg} / \mathrm{kg}$ body weight, 1 time in the beginning of the experiment), or diethylnitrosamine (DEN, Sigma-Aldrich; $50 \mathrm{mg} / \mathrm{kg}$ body weight, one time/ week) in $40 \%$ olive oil for 16 or 27 weeks, respectively. For 4'-( $p$-toluenesulfonylamido)-4-hydroxychalcone (TSAHC) [25] administration [50 mg/kg in $40 \%$ dimethylsulfoxide (DMSO)], mice were injected intraperitoneally for 16 weeks (every 2 or 3 days per week). TSAHC injection was started either at week 0 (DEN + TSAHC1 group) or week 10 (DEN + TSAHC2 group) for overall 27 weektreatment for DEN-treated liver cancer model. Alternatively, age-matched (2-week-old) WT and Tm $4 s f 5^{-1-}$ mice $(n \geq 5)$ were injected intraperitoneally with or without DEN $(25 \mathrm{mg} /$ $\mathrm{kg}$ body weight) at day 0 . TSAHC was also injected intraperitoneally twice a week ( $5 \mathrm{mg} / \mathrm{kg}$ body weight). After 45 weeks, the mice were sacrificed for analyses. The mice were euthanized with ether, and the liver and spleen tissues were resected. One piece of liver tissue was immediately frozen in liquid $\mathrm{N}_{2}$, whereas a second piece was embedded in paraffin or alternatively used for primary hepatocyte preparation. Plasma samples were also collected for plasma parameter analyses.

\section{Western blot analysis}

Sub-confluent cells in normal culture media were transfected or infected with control or specific siRNA, shRNA vectors, or virus encoding the indicated molecules for 48 or $24 \mathrm{~h}$, respectively, in the presence of vehicle or TSAHC [26] treatment. Animal liver tissues were harvested for whole-cell or tissue extraction using a modified RIPA buffer, as previously described [17, 21]. The primary antibodies (generally at 1:1000 dilution) used included antibodies against laminins, $\mathrm{pY}^{397}$ FAK, MICA/B (Abcam, Cambridge, UK), p-ERKs, ERKs (Cell Signaling Technology. Danvers, MA, USA), $\mathrm{pY}^{705}$ STAT3 (Millipore, Billerica, MA, USA), $\beta$-actin, $\alpha$-tubulin, SLAMF7 (CS1), MICA/B, STAT3, pY ${ }^{577}$ FAK (Santa Cruz Biotechnology, Santa Cruz, CA, USA), FAK (BD Transduction Laboratories, Bedford, MA, USA), collagen I (Acris Antibodies, Herford, Germany), and TM4SF5 [17]. The TM4SF5 ${ }_{\text {C-ter }}$ (epitope region of RKKQDTPH ${ }^{197}$ ) antibody was custom designed (Pro-Sci, Poway, CA, USA).

\section{Natural killer cell cytotoxicity assay}

Evaluation of NK cell cytotoxic activity was performed using a lactate dehydrogenase (LDH) cytotoxicity assay kit (Cytotoxicity Detection Kit Plus, Roche). Human HCC cell lines (Huh7 or HepG2 cells with endogenous TM4SF5 expression; target cancer cells) were seeded $\left(0.5 \times 10^{4}\right.$ cells/ well; $50 \mu \mathrm{L}$ ) into a 96-well plate in triplicate with assay medium (RPMI 1640 with 1\% FBS). Human NK92 cells 
pre-treated with IL-2 (effector cells) were adjusted to a concentration of $1 \times 10^{6}$ cells $/ \mathrm{mL}$ in assay medium, and then two-fold dilutions of the NK cells were prepared $\left(1 \times 10^{6}\right.$, $0.5 \times 10^{6}, 0.25 \times 10^{6}$, and $0.125 \times 10^{6}$ cells $\left./ \mathrm{mL}\right)$. NK cell suspension was pipetted into the wells of the 96-well plate in triplicate at effector $(\mathrm{E})$ to target $(\mathrm{T})$ cell ratios $(\mathrm{E}: \mathrm{T})$ of 1.25:1, 2.5:1, 5:1, and 10:1 to see the TM4SF5-dependent effects. Cells were incubated in the 96-well plate for $4 \mathrm{~h}$ at $37{ }^{\circ} \mathrm{C}$ in $5 \% \mathrm{CO}_{2}$. LDH released from the dead target cells was measured using an ELISA reader at 492-690 nm. Percentage (\%) of cytotoxicity was calculated as follows: [LDH (effector-target cell mix) - LDH (effector cell) - LDH (target cell low control)]/[LDH (target cell high control) - LDH (target cell low control)] $\times 100$. After co-culturing, nonadherent NK cells were collected and centrifuged at $150 \times g$ for $5 \mathrm{~min}$ to remove the target cancer cell debris. Quantitative RT-PCR was then performed to analyze the ligand or receptor mRNA levels of the hepatocytes or NK92 cells, respectively.

\section{Murine splenic and intrahepatic immune cell analysis}

Mouse spleens and livers were dissected, and cell suspensions were generated by mechanical disruption through 70or 100- $\mu \mathrm{m}$ nylon mesh filters, respectively. Immune cells were isolated from the spleens and suspended in RPMI 1640 (with 2\% FBS). The immune cell suspension was then centrifuged at $480 \times g$ for $8 \mathrm{~min}$, and the supernatant was removed. The cell pellets were resuspended in ammoniumchloride-potassium (ACK) buffer (RBC lysis buffer, Thermo Fisher Scientific, Cat. No: A1049201) and incubated for 5 min at $37{ }^{\circ} \mathrm{C}$. Next, the cell suspension was centrifuged at $480 \times g$ for $8 \mathrm{~min}$, and the supernatant was removed. The cell pellets were washed twice using FACS staining buffer (BD Bioscience, Cat. No: 554657) and centrifuged at $480 \times g$ for 8 min. The cell pellet suspensions with the staining buffer were then incubated with Fc Block (BD Bioscience, Cat. No: 564219) at $4{ }^{\circ} \mathrm{C}$ for 15 min to block the lymphocyte $\mathrm{Fc} \gamma \mathrm{II} / \mathrm{III}$ receptors. Intrahepatic immune cells were enriched using a $37.5 \%$ Percoll (GE Healthcare) gradient. Erythrocytes were lysed with red blood cell (RBC) lysis buffer (Invitrogen). To assess IFN- $\gamma$, perforin 1 (PRF1), and granzyme B (GZMB) expression, lymphocytes were incubated with $5 \mathrm{ng} / \mathrm{mL}$ phorbol 12-myristate 13-acetate (PMA, Sigma), $500 \mathrm{ng} / \mathrm{mL}$ ionomycin (Sigma), and $10 \mathrm{ng} / \mathrm{mL}$ monensin (Sigma), respectively, for $4 \mathrm{~h}$ at $37{ }^{\circ} \mathrm{C}$ in $5 \% \mathrm{CO}_{2}$. Lymphocyte FcgII/III receptors were blocked with Fc Block (BD Bioscience), and surface antigens were stained with conjugated and biotinylated monoclonal antibodies (mAbs). Splenic or intrahepatic immune cells were then fixed and permeabilized using Cytofix/Cytoperm (BD Bioscience), and intracellular antigens were detected using conjugated and biotinylated mAbs at $4{ }^{\circ} \mathrm{C}$ for $30 \mathrm{~min}$. Phycoerythrin (PE)-conjugated anti-human PRF1 allophycocyanin (APC)conjugated anti-mouse GZMB, PE-conjugated anti-mouse PRF1 fluorescein isothiocyanate (FITC)-conjugated antimouse CD45, PE-conjugated anti-mouse CD4, APC/Cy7conjugated anti-mouse $\mathrm{CD} 3$, FITC-conjugated anti-mouse CD8a (BioLegends), BV421-conjugated anti-mouse CD8a, and BV421-conjugated anti-mouse NK1.1 antibodies (BD Bioscience) were used for staining. Flow cytometry analysis was performed on a FACS LSR Fortessa X-20 (BD) and analyzed with FlowJo version 10.6.1. Graphic data were presented the mean \pm standard error of the mean (SEM).

\section{Immunohistochemistry and tissue staining}

Liver tissues from human liver cancer patients, $\mathrm{Tg}^{\mathrm{TM} 4 \mathrm{SF} 5}$ FVB/N mice, or C57BL/6 (WT or $T m 4 s f 5^{-1-} \mathrm{KO}$ ) mice treated with or without DEN and in the absence or presence of TSAHC were processed for immunohistochemistry. Liver sections were fixed with $3.7 \%$ formaldehyde and embedded in paraffin. The fixed liver sections were deparaffinized and rehydrated. Antigen retrieval was performed with heatinduced epitope retrieval (HIER) using sodium citrate buffer ( $\mathrm{pH}$ 6.0). Quenching and blocking were performed using $3 \% \mathrm{H}_{2} \mathrm{O}_{2}$ in distilled water and $1 \%$ normal goat serum in phosphate-buffered saline (PBS). Antigens were stained using the avidin-biotin complex (ABC) method (VECTASTAIN Elite ABC HRP Kit, Vector) and were detected using 3,3'-diaminobenzidine (DAB) stain (Vector). Antibodies against TM4SF5 [17], collagen I (Acris Antibodies), pY ${ }^{705}$ STAT3 (Cell Signaling Technology), normal rabbit or mouse IgG, $\alpha$-SMA (Sigma-Aldrich), $\alpha$-fetoprotein (AFP), CD34, Ki67, laminins (Abcam), $\alpha$-L-fucosidase [FUCA (AFU)], and laminin $\gamma 2$ (Santa Cruz Biotechnology) were used for immunostaining. Ten random images per slide were saved using a digital slide scanner (MoticEasyScan, Motic, British Columbia, Canada). The tissues were also processed with Masson's trichrome as well as hematoxylin and eosin stains, as previously described [27].

\section{Polymerase chain reaction}

Total RNA from animal liver tissues or cells were isolated using TRIzol reagent (Invitrogen), and total RNA isolated from the samples was treated with ReverTra Ace qPCR RT 
Master Mix (TOYOBO) with gDNA Remover to generate complementary DNA (cDNA). The cDNA was subjected to RT-PCR using the Dream Taq Green PCR Master Mix (Thermo Scientific, San Jose, CA, USA). Quantitative RT-PCR was prepared with LaboPass EvaGreen Q Master (Cosmo Genetech, Seoul, Korea) and performed with the CFX Connect Real-Time PCR Detection System (Bio-Rad, Hercules, CA, USA). The mRNA levels were normalized against $18 \mathrm{~S}$ ribosomal RNA, using the ddCq method. The CFX Maestro Software (Sunnyvale, CA, USA) was used to analyze the data. Primers were purchased from Cosmo Genetech (Seoul, Korea). The primer sequences are shown in Table 1.

\section{Analysis of public RNA expression data}

The mRNA expression levels of some genes were searched in the public Gene Expression Omnibus (GEO, https:// www.ncbi.nlm.nih.gov/geo/) database (accession numbers GSE6764, GSE14520, and GSE76427). The mRNA expression levels were extracted using the built-in function, 'Analyze with GEO2R'. The specific identification numbers for TM4SF5 and SOCS1 were 8003939 and 7999423 from GSE6764, respectively, and TM4SF5 and LAMC2 were ILMN 2167808 and ILMN 1653824 from GSE76427, respectively. Public identification numbers of GSE14520 were for TM4SF5 (ID No. 4507538), SLAMF7 (12711663), MICA (4557750), ULBPI (13376825), and ULBP2 (13376823). The available mRNA expression levels for the indicated groups were further analyzed for comparison. The
Table 1 The primer sequences for RT-PCR or q-PCR in the current study

\begin{tabular}{|c|c|c|}
\hline \multirow[t]{2}{*}{ Gene name } & \multicolumn{2}{|l|}{ Sequence } \\
\hline & Forward $\left(5^{\prime} \rightarrow 3^{\prime}\right)$ & Reverse $\left(5^{\prime} \rightarrow 3^{\prime}\right)$ \\
\hline \multicolumn{3}{|l|}{ Human } \\
\hline TM4SF5 & CTTGCTCAACCGCACTCTAT & ATCCCACACAGTACTATCTCCA \\
\hline hSLAMF6 & GTCCAGAAATCCACGTGACTAA & GTAAGAGCCTGTGTCTTCCATC \\
\hline hSLAMF7 & TGGGTCTGCAGAGCAATAAG & CAGGGCCTTCCAGGTATAAAT \\
\hline hMICA & CCTTGGCCATGAACGTCAGG & CCTCTGAGGCCTCGCTGCG \\
\hline$h M I C B$ & ACCTTGGCTATGAACGTCACA & CCCTCTGAGACCTCGCTGCA \\
\hline hULBP1 & TTTCCTTAAAGGGCAACTGCT & AGGAACTGCCAAGATCCTCT \\
\hline hULBP2 & САTTACTTCTCAATGGGAGACTGT & TGTGCCTGAGGACATGGCGA \\
\hline hULBP3 & CTGATGCACAGGAAGAAGAG & TATGGCTTTGGGTTGAGCTAA \\
\hline hULBP5 & GACTCGCGTGACTTTACCTATC & GGCTGCGCCGTTATTTATTG \\
\hline hCD111(Nectin-1) & ATCCTGCTGGTGTTGATTGT & TGCTTCTTGGTGCTGTAGTC \\
\hline hCD112(Nectin-2) & TTCCCTGGATCTAGAGGATGAG & CTTTGCCCTGGTAGGAATCA \\
\hline hCD113(Nectin-3) & TTGCTAGAGGAAGGCGAATTAC & ACCGAAACTTCAGGAGCATAC \\
\hline$h C D 155(P V R)$ & AGGTATCCATCTCTGGCTATGA & CCATGGTCGTGCTCCAATTA \\
\hline hCADM1 & GCTTCTGCTGTTGCTCTTCT & CTCGATCACTGTCACGTCTTTC \\
\hline$h K l r k l(N K G 2 D)$ & CAGCAAAGAGGACCAGGATTTA & GTTAGTAGGTTGGGTGAGAGAATC \\
\hline hPRF1 & CCCAGTGGACACACAAAGGTT & TCGTTGCGGATGCTACGAG \\
\hline$h G Z M B$ & CTTCCTGATACGAGACGACTTC & CGGCTCCTGTTCTTTGATATTG \\
\hline$h C D 107 a$ & CGTCAGCAGCAATGTTTATGG & CATGTTCTTAGGGCCACTCTT \\
\hline$h T N F-\alpha$ & $\begin{array}{l}\text { GAGTGACAAGCCTGTAGCCCATGT } \\
\text { TGTAGCA }\end{array}$ & $\begin{array}{l}\text { GCA ATG ATCCCAAAGTAGACC } \\
\text { TGCCCAGACT }\end{array}$ \\
\hline hFasL & CATTTAACAGGCAAGTCCAACTC & CACAAGGCCACCСТTCTTAT \\
\hline hTNFSF10(TRAIL) & CAGAGAGTAGCAGCTCACATAAC & CCTTGATGATTCCCAGGAGTTT \\
\hline \multicolumn{3}{|l|}{ Mouse } \\
\hline$T m 4 s f 5$ & GTCTTCTCCTCCGCCTTTG & GGTAGTCCCACTTGTTGTCTATT \\
\hline$F 4 / 80$ & ACCACAATACCTACATGCACC & AAGCAGGCGAGGAAAAGATAG \\
\hline Mcp-1 & TTAAAAACCTGGATCGGAACCAA & GCATTAGCTTCAGATTTACGGGT \\
\hline$T n f-\alpha$ & СCCTCACACTCAGATCATCTTCT & GCTACGACGTGGGCTACAG \\
\hline Il-6 & GAGGATACCACTCCCAACAGACC & AAGTGCATCATCGTTGTTCATACA \\
\hline$C d 34$ & CAGGAGAAAGGCTGGGTGAA & GTTGTCTTGCTGAATGGCCG \\
\hline Ki67 & AAAGGCGAAGTGGAGCTTCT & TTTCGCAACTTTCGTTTGTG \\
\hline Gapdh & GACATGCCGCCTGGAGAAAC & AGCCCAGGATGCCCTTTAGT \\
\hline
\end{tabular}


A
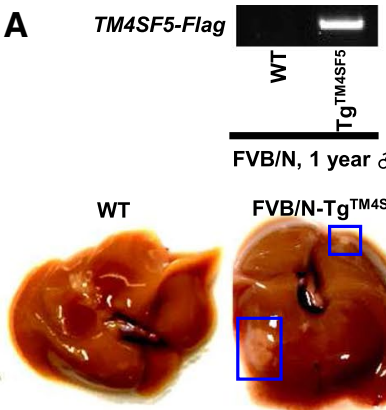

FVB/N, 1 year $\delta$

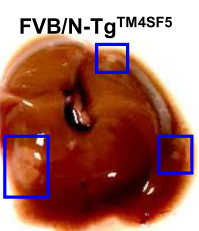

B

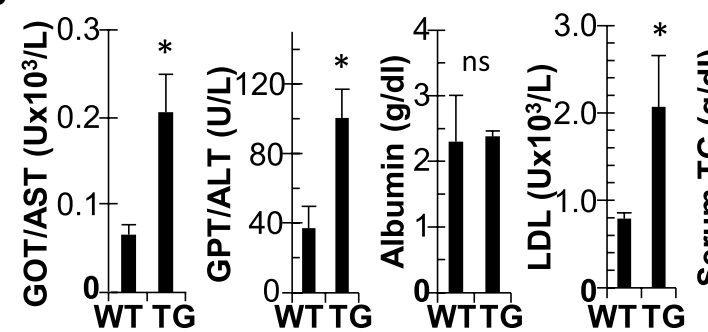

C

Ki67

Gapdh

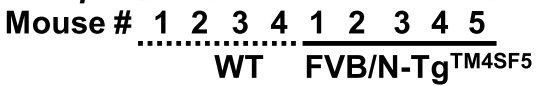

D

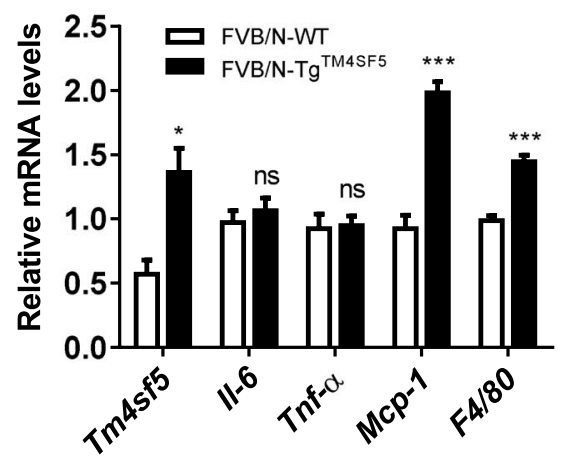

E

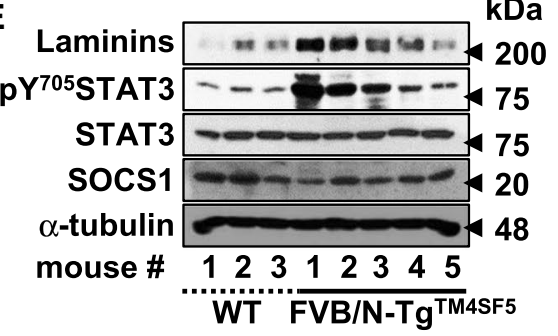

$\mathbf{F}$

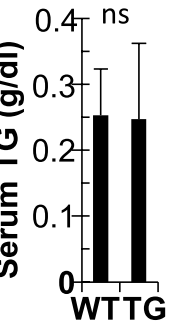

WTTG

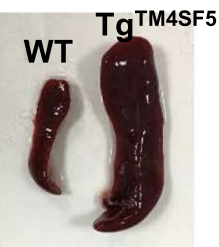

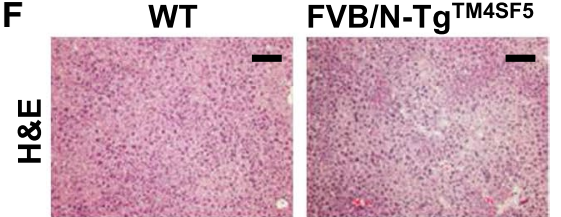
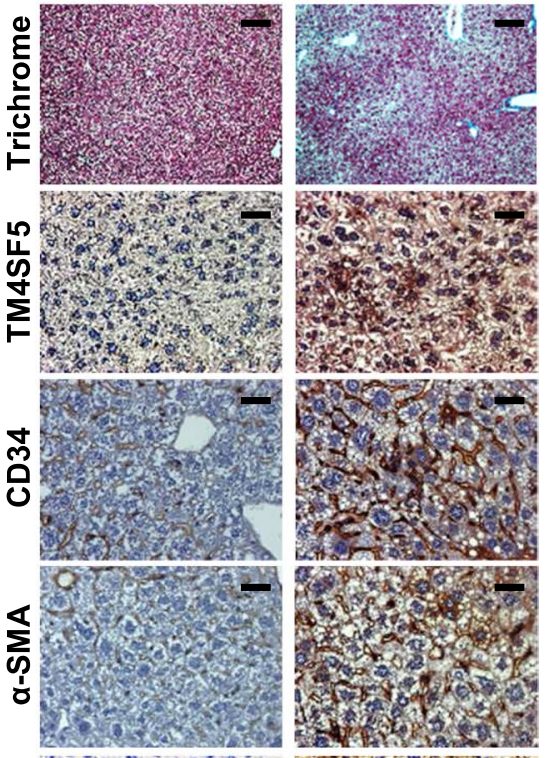

(2)
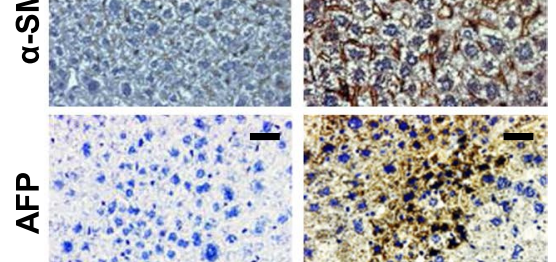

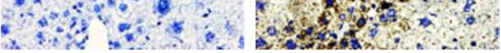
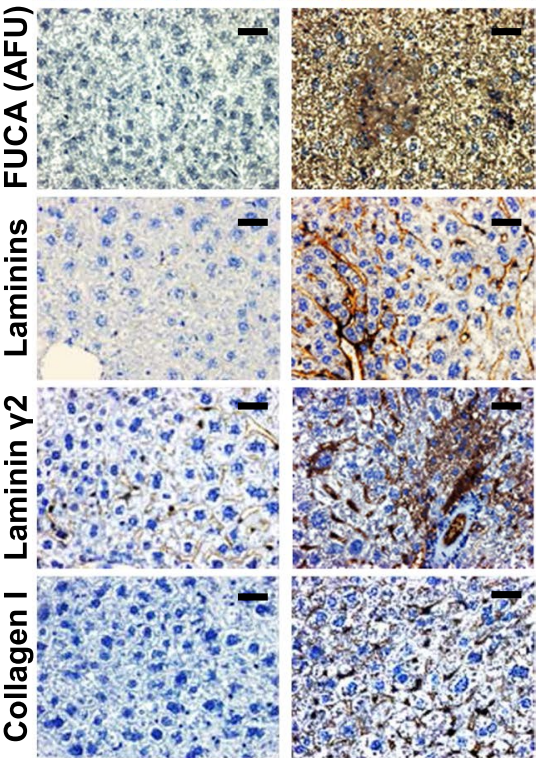

Mann-Whitney $U$ test was applied for statistical analysis using GraphPad Prism 7.0. A box-and-whisker plot was drawn using GraphPad Prism 7.0.

\section{Study approval}

Human liver tissues were collected after obtaining informed consent from the liver cancer patients at the Seoul National University under institutional review board (IRB)-approved protocols (Seoul, Korea). All animal procedures were performed in accordance with the Seoul 
4Fig. 1 TM4SF5-mediated hepatic carcinogenesis in TM4SF5-transgenic mice. A Liver nodules in 1-year-old FVB/N-Tg ${ }^{\text {TM4SF5 }}$ mice $(n=2 / 7)$ compared with age-matched wild-type (WT) mice $(n=0 / 7)$. $\mathrm{Tg}^{\text {TM4SF5 }}$ mice showed enlarged spleens compared with age-matched WT mice. B The livers of 1-year-old FVB/N-Tg ${ }^{\text {TM4SF5 }}$ mice were examined for aspartate aminotransferase (AST), alanine aminotransferase (ALT), low-density lipoprotein (LDL), albumin, and serum triglyceride (TG) levels and were compared with those of age-matched WT mice. C, D The mRNA levels of the livers of 1-year-old FVB/N$\mathrm{Tg}^{\text {TM4SF5 }}$ and age-matched WT mice were analyzed for the indicated molecules. For statistical significance, $*$ and $* * *$ represent $p \leq 0.05$ and $p \leq 0.001$, respectively, and ns indicates no significance, following Student's $t$ tests. E, F The livers of 1 -year-old FVB/N-Tg ${ }^{\text {TM4SF5 }}$ and age-matched WT mice were processed for immunoblot $(\mathbf{E})$ and immunohistochemical $(\mathbf{F})$ analyses. The livers of $\mathrm{Tg}^{\mathrm{TM} 4 \mathrm{SF} 5}$ mice showed enhanced expression of hepatocellular carcinoma markers, including CD34, $\alpha$-fetoprotein (AFP), and $\alpha$-L-fucosidase [FUCA (AFU)], in addition to $\mathrm{pY}^{705}$ STAT3 and extracellular matrix factors, including collagen I, laminin $\gamma 2$, and laminins. Data shown represent three isolated experiments

National University Laboratory Animal Maintenance Manual and were approved by the IRB of the Institute of Laboratory Animal Resources Seoul National University (SNU-IACUC) (SNU-140423-11-7, SNU-130911-2-3, SNU-170920-9, SNU-190122-6-3).

\section{Statistics}

Statistical analyses were performed using Prism Software (GraphPad 7.0, La Jolla, CA, USA). Two-way analysis of variance (ANOVA), ordinary one-way ANOVA, an unpaired, one-tailed Mann-Whitney $U$ test, or unpaired, two-tailed Student's $t$ tests were performed to determine statistical significance. A $p$ value $<0.05$ was considered statistically significant.

\section{Results}

\section{TM4SF5 mediated hepatic carcinogenesis}

Because C57BL/6-Tg ${ }^{\text {TM4SF5 }}$ mice exhibited nonalcoholic steatohepatitis (NASH)-associated fibrotic livers [24], we determined whether TM4SF5 overexpression in a disease-susceptible mouse strain (i.e., FVB/N [23]) caused an enhanced malignant cancer phenotype. The livers of 1-year-old FVB/N-Tg ${ }^{\text {TM4SF5 }}$ mice were analyzed to test this hypothesis. Out of seven mice, we found two with hepatic nodules, suggesting hepatic carcinogenesis. Interestingly, the $\mathrm{Tg}^{\mathrm{TM} 4 \mathrm{SF} 5}$ mice also exhibited enlarged spleens compared with age-matched wild-type (WT) mice (Fig. 1A). The FVB/N-Tg ${ }^{\text {TM4SF5 }}$ mice showed higher aspartate aminotransferase (AST), alanine aminotransferase (ALT), and low-density lipoprotein (LDL) levels with comparable levels of albumin and serum triglycerides (TGs) compared with age-matched WT mice (Fig. 1B). Furthermore, CD34 and Ki67 mRNA levels were higher in the livers of $\mathrm{FVB} / \mathrm{N}$ $\mathrm{Tg}^{\text {TM4SF5 }}$ mice than in age-matched WT mice (Fig. 1C), indicating cell proliferation. Interestingly, liver monocyte chemoattractant protein 1 [Mcp- 1 (chemokine ligand 2, Ccl2)] and F4/80 mRNA levels were also elevated in the $\mathrm{Tg}^{\mathrm{TM} 4 \mathrm{SF} 5}$ mice compared with age-matched WT mice, indicating immune system involvement (Fig. 1D). Indeed, serum MCP-1 was reported to be positively correlated with $\alpha$-fetoprotein (AFP) levels, suggesting it as another tumor marker in HCC [28]. The levels of laminins and $\mathrm{pY}^{705}$ STAT3 (Fig. 1E), collagen I, $\alpha$-smooth muscle actin (SMA), laminins, laminin $\gamma 2$, and TM4SF5 expression levels were elevated in FVB/N-Tg ${ }^{\mathrm{T}}$ M4SF5 mice compared with age-matched WT mice, in addition to CD34, AFP, $\alpha$-L-fucosidase [FUCA (AFU)], which are HCC markers [29-31], hepatic damage, and collagen I deposition (Fig. 1F). These observations support the hypothesis that the livers of FVB/N Tg ${ }^{\mathrm{TM} 4 \mathrm{SF} 5}$ mice become cancerous. Moreover, immunostaining of liver tissues from HCC patients showed substantially elevated levels of TM4SF5, laminins, collagen I, and $\mathrm{pY}^{705}$ STAT3 in peritumoral (presumably with NASH and fibrosis phenotypes) and tumor regions compared with normal regions (Fig. 2A). Importantly, laminin-positive cells were predominantly appeared to be hepatocytes with large cytosolic spaces, which were different from collagen I-positive cells (Fig. 2A), suggesting a role of TM4SF5-induced laminins in hepatocytes during hepatic carcinogenesis. In addition, public Gene Expression Omnibus (GEO) data analysis showed that TM4SF5 expression was highly elevated in HCC patients, and these elevated levels were negatively correlated with SOCS1 expression in both cirrhosis and HCC patients compared with the normal population (Fig. 2B). Further, increased TM4SF5 expression was also positively correlated with enhanced $L A M C 2$ expression (Fig. 2C). Indeed, STAT3 suppression in the primary hepatocytes of $\mathrm{CCl}_{4}$-induced cirrhotic livers (16week treatment) decreased collagen I and laminin expression in addition to STAT3 and FAK phosphorylation, as downstream effectors of TM4SF5 (Fig. 2D). These findings suggest that TM4SF5/STAT3-mediated expression of ECM factors may be involved in fibrosis/cirrhosis and subsequent hepatic carcinogenesis. 
Fig. 2 Clinical relevance of TM4SF5-mediated STAT3 activity, extracellular matrix production, and hepatic cancer. A Human liver cancer tissues were processed for immunohistochemical analysis and showed higher levels of TM4SF5, $\mathrm{pY}^{705}$ STAT3, collagen I, and laminins, compared with normal surrounding liver tissues. B, C Gene expression profiles of liver cirrhosis [normal (Nor) vs. cirrhosis (Cir) vs. HCC] from GSE6764 were analyzed and showed TM4SF5-positive cirrhosis $(p=0.055)$ and HCC $(p=0.011)$ and SOCS1-reduced cirrhosis and HCC $(p=0.013)$ groups. $p$-values were calculated by the Dunnett's test and ordinary one-way ANOVA. Other analyses of hepatocellular carcinoma (HCC) (normal vs. HCC) from GSE76427 showed TM4SF5-positive $(p=0.010)$ and LAMC2-promoted HCC $(p=0.016)$ groups compared with normal groups. $p$-values were calculated by the MannWhitney $U$ test. D Primary hepatocytes were prepared from mice treated with $\mathrm{CCl}_{4}$ for 16 weeks as an animal cirrhosis model and were transfected with STAT3-specific or scrambled control siRNA for $48 \mathrm{~h}$ before harvesting whole-cell extracts for immunoblot analysis. Data shown represent three independent experiments
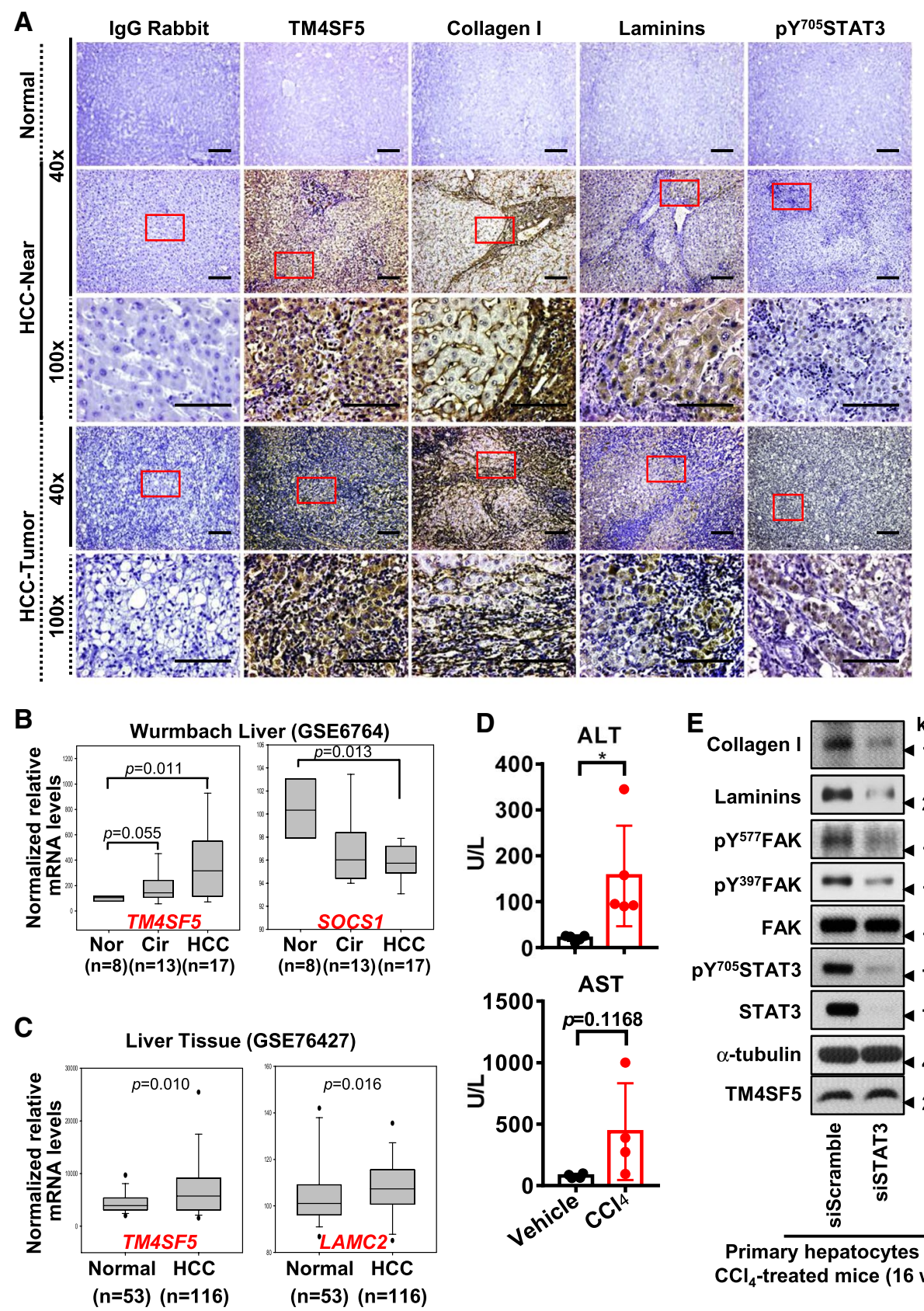

\section{TM4SF5 inhibitor abolished diethylnitrosamine-induced hepatic carcinogenesis}

Next, we investigated the roles of the immune system in TM4SF5-mediated hepatic carcinogenesis using $T m 4 s f 5^{-1-}$ and age-matched WT mice treated with or without diethylnitrosamine (DEN) in the absence or presence of TM4SF5 inhibitor 4'-( $p$-toluenesulfonylamido)4-hydroxychalcone (TSAHC) treatment. The mice were treated once with DEN and twice a week with TSAHC. After 45 weeks, the livers of the mice were analyzed, as depicted in the scheme (Fig. 3A). The livers of the mice exhibited dramatic differences in nodule formation depending on TM4SF5 expression and function (i.e., activity). Age-matched WT and knockout mice without DEN treatment did not form liver nodules. However, WT mice treated with DEN showed dramatic nodule formations, which was abolished by additional TSAHC treatment (Fig. 3B). Indeed, 
$T m 4 s f 5^{-1-}$ mice did not show substantial nodule formation, with being similar in the DEN-treated WT mice with TSAHC treatment (Fig. 3B). Quantitative analysis in terms of the number of tumors larger than $0.1 \mathrm{~mm}$ in diameter, tumor load (as the sum of tumor diameter of all tumors with diameters $>1.0 \mathrm{~mm}$ ), and the maximal tumor size resulted in greater values for DEN-treated WT mice than DENtreated knockout mice and DEN/TSAHC-treated WT mice, although the change tendencies were statistically significant $(p=0.0470)$ or insignificant ( $p=0.0757-0.1910)$ (Fig. 3C). Unlike the FVB/N-Tg ${ }^{\mathrm{TM} 4 \mathrm{SF} 5}$ mice, DEN-treated WT mice did not have significantly larger spleen sizes compared with DEN-treated knockout mice and DEN/TSAHC-treated WT mice ( $p=0.4065$, Fig. 3D).

Hematoxylin and eosin (H\&E) staining and immunohistochemical analyses of the liver tissues showed dramatically different tumor lesions among the mice. More aggressive tumor lesions were shown in the DEN-treated WT mice by positive Ki67 and AFP staining, whereas DEN-treated knockout and DEN/TSAHC-treated WT mice showed fewer tumors with less positive or negative Ki67 and AFP staining (Fig. 3E). Interestingly, we found significant formation of noninfectious granulomatous inflammation (with immune cell infiltrates) near the portal tracks with positive AFP staining in the DEN-treated WT mice, which was abolished by additional TSAHC treatment (Fig. 3F). These observations indicate that TM4SF5 activation may be involved in the inflammation during DEN-induced hepatic carcinogenesis and that inhibition of TM4SF5 by TSAHC may interfere with the DEN-mediated effects.

\section{Natural killer cells were inhibited by TM4SF5 during diethylnitrosamine-induced hepatic carcinogenesis}

Because the hepatic granuloma-like lesions within the DEN-induced, AFP-positive tumors exhibited immune cell infiltration, we investigated the potential mechanism of the immune cells in carcinogenesis. First, we used immunostaining and FACS to analyze the population of splenic immune cells for $\mathrm{CD}^{+} / \mathrm{CD}^{+}$(T-helper cells, Th), $\mathrm{CD}^{+}{ }^{+}$ $\mathrm{CD}^{+}$(cytotoxic $\mathrm{T}$ cells), and $\mathrm{CD}^{-} / \mathrm{NK} 1.1^{+}$(NK cells) cell markers (Fig. 4A). Splenic CD4 ${ }^{+}$Th-cell populations were not significantly different between vehicle- and DEN-treated WT or KO mice; however, DEN/TSAHC-treated WT mice showed significantly $\left(p=0.0331\right.$ ) lower $\mathrm{CD} 4^{+}$Th-cell populations compared with DEN-treated WT mice (Fig. 4B, left).
$\mathrm{CD} 8^{+} \mathrm{T}$-cell populations were not differential between vehicle-treated WT and KO mice ( $p=0.9155)$, and significantly increased in KO mice upon DEN treatment $(p=0.0362)$ or in DEN-treated WT mice upon further TSAHC treatment $(p=0.0376)$ (Fig. 4B, middle). Therefore, $\mathrm{CD}^{+}{ }^{+} \mathrm{Th}-$ and $\mathrm{CD} 8^{+} \mathrm{T}$-cell populations appeared not to be significantly involved in the immune effects during TM4SF5 expression (e.g., WT vs. KO) but in TM4SF5 function/activity-dependent carcinogenesis (e.g., WT-DEN vs. WT-DEN/TSAHC). However, consistent with the tumor load patterns, DENtreated WT mice showed a slight but insignificant reduction in the population of NK cells compared with vehicle-treated WT mice $(p=0.1195)$, and DEN-treated KO mice and DEN/ TSAHC-treated WT mice exhibited slight but statistically insignificant recoveries in the splenic NK cell population ( $p=0.1510$ or 0.1528 , respectively), suggesting a possible involvement (though insignificant presumably due to smaller sample numbers) of NK cells in response to TM4SF5 blockade with TSAHC during DEN-induced carcinogenesis (Fig. 4B, right).

We also examined the intrahepatic immune cell populations via immunostaining and FACS for $\mathrm{CD}^{+} / \mathrm{CD} 4^{+}$Thcells, $\mathrm{CD}^{+} / \mathrm{CD}^{+}{ }^{+} \mathrm{T}$-cells, and $\mathrm{CD}^{-} / \mathrm{CD} 45^{+} / \mathrm{NK} 1.1^{+} \mathrm{NK}$ cells (Fig. 4C). Unlike splenic CD4 ${ }^{+}$Th-cells, hepatic CD4 ${ }^{+}$ Th-cells were reduced in DEN-treated WT mice and were not recovered by TSAHC treatment or Tm $4 s f 5$-knockout (Fig. 4D, left). Furthermore, hepatic CD8 ${ }^{+}$T-cell populations were increased in WT mice upon DEN treatment $(p=0.0076)$, which were not changed by TSAHC treatment or Tm4sf5-deficiency (Fig. 4D, middle). Additionally, DEN-treated WT mice showed higher populations of NK cells than vehicle-treated WT mice $(p=0.0435)$, which was similar to the levels of DEN-treated KO mice $(p=0.4295)$ or DEN/TSAHC-treated WT mice ( $p=0.9655$, Fig. 4D, right). Therefore, intrahepatic immune cells, including Th, cytotoxic T, and NK cell populations, did not appear to be correlated with the TM4SF5-dependent carcinogenesis in DEN-treated mice. However, splenic NK cell numbers might still have roles in DEN-induced carcinogenesis, depending on TM4SF5 activity, although their effects were not statistically insignificant presumably due to small sample numbers (Fig. 4B).

We therefore analyzed further intrahepatic NK cell activity by immunostaining for perforin, granzyme, and cytokine IFN- $\gamma$. Although DEN-treated WT mice exhibited lower levels of IFN- $\gamma$ and granzyme than vehicle-treated WT mice, their levels were not significantly different by additional TSAHC treatment ( $p=0.6920$ and $p=0.3897$, respectively) 


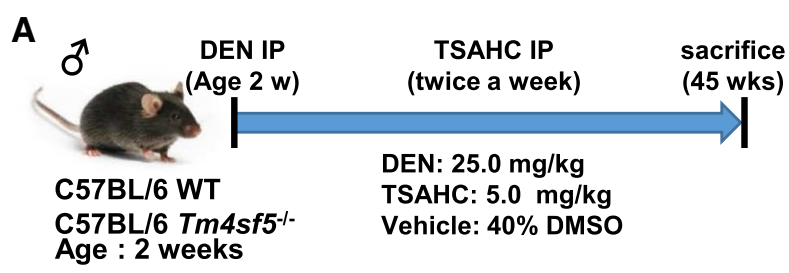

B
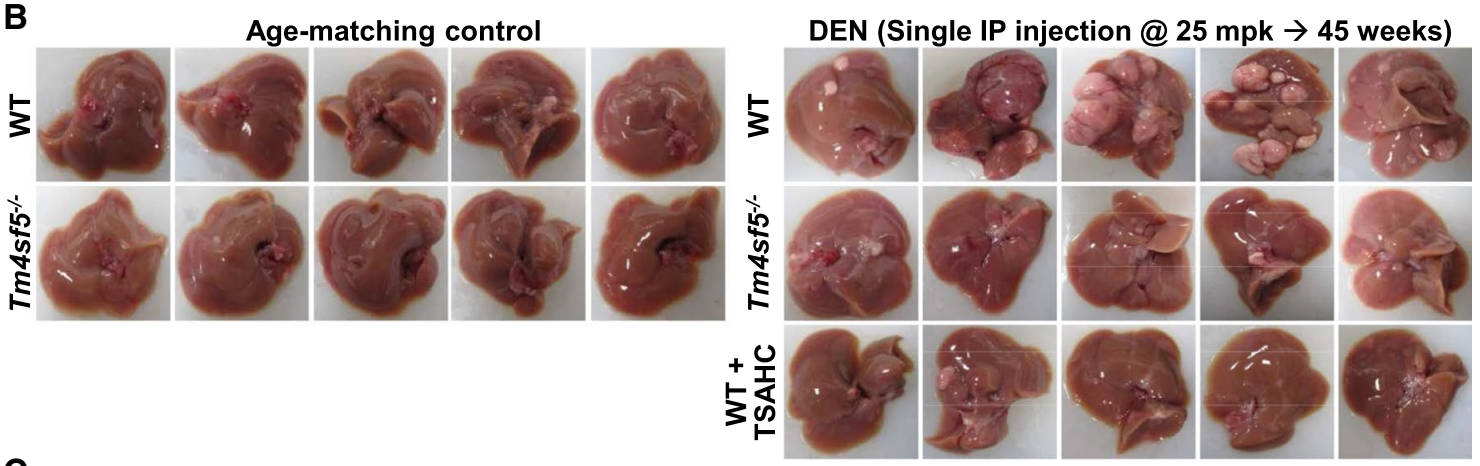

C
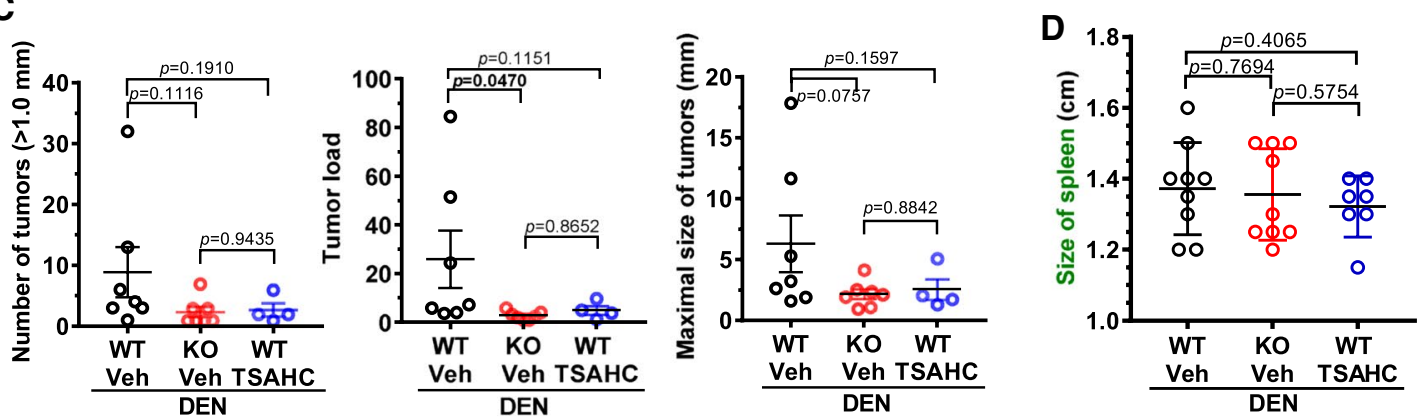

E
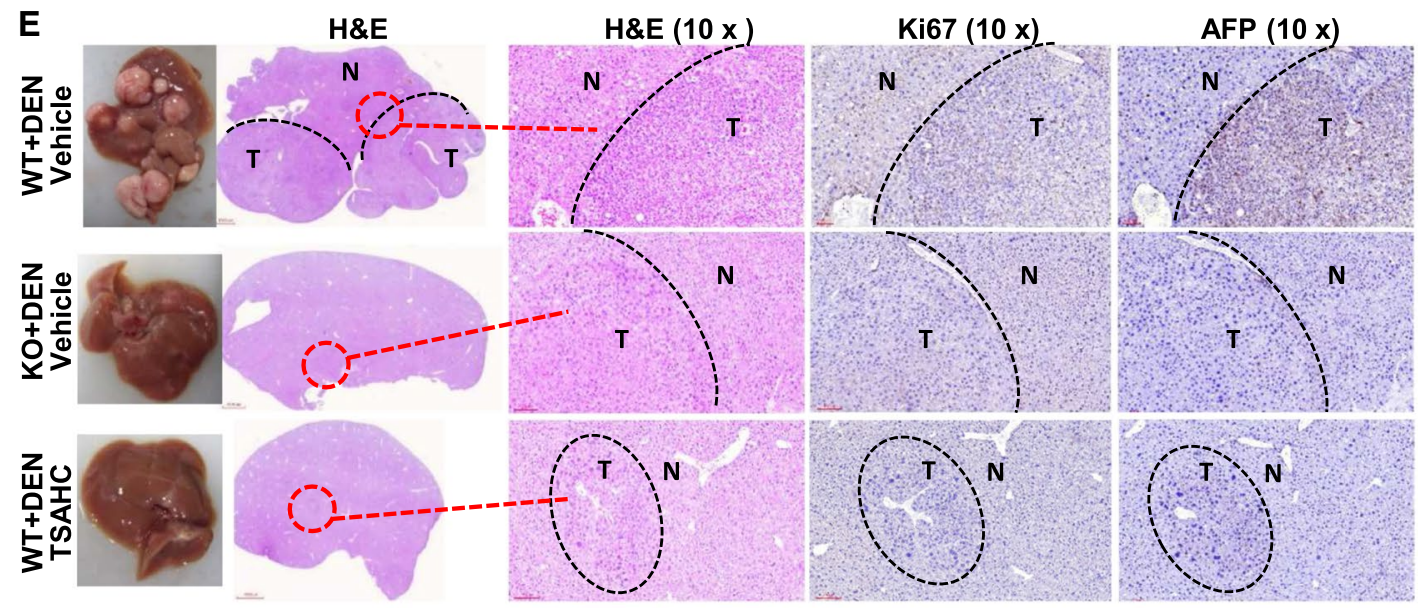

F

WT + DEN + Vehicle
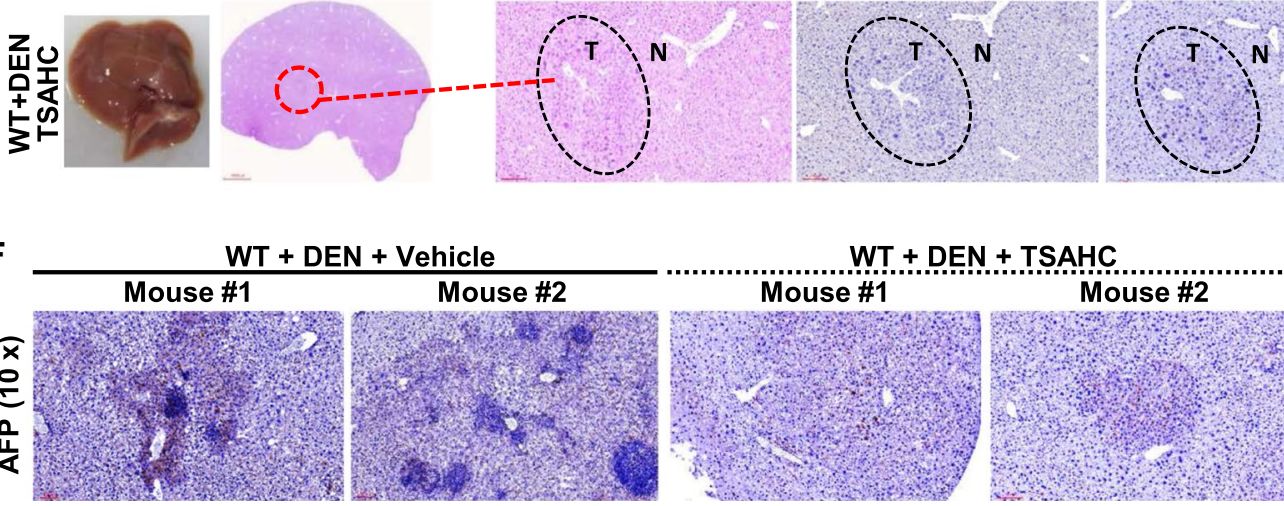

$W T+$ DEN + TSAHC

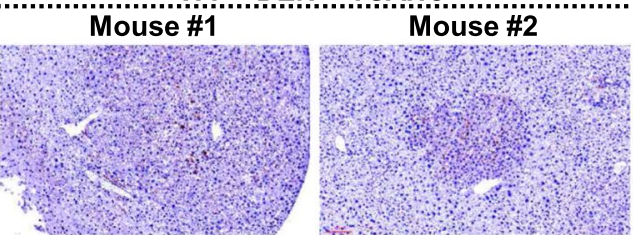


4Fig. 3 TM4SF5 expression and activity-dependent granulomatous inflammation during diethylnitrosamine-induced hepatic carcinogenesis. A Age-matched WT or Tm $4 s f 5^{-1-}$ male C57BL/6 mice (2 weeks old) were intraperitoneally injected once with diethylnitrosamine (DEN, $25 \mathrm{mg} / \mathrm{kg}$ body weight) and twice per week with 4'-( $p$-toluenesulfonylamido)-4-hydroxychalcone (TSAHC, $5.0 \mathrm{mg} / \mathrm{kg}$ body weight) for 45 weeks. After 45 weeks on a normal diet ad libitum, the mice were sacrificed for analysis. Age-matched WT and knockout mice $(n=6)$, DEN-treated WT and knockout mice $(n=7)$, and DEN/TSAHC-treated WT mice $(n=5)$ were examined in parallel. B Five representative liver images were shown for each group. $\mathbf{C}$ Tumors with a diameter larger than $1.0 \mathrm{~mm}$ were counted, and the largest tumor in each animal was determined. Age-matched WT and knockout animals without DEN treatment did not form any tumors. One outlier (second, left) from the DEN/TSAHC-treated WT group was not included in the graphs. $p$-values were calculated by Sidak's test and one-way ANOVA. A $p$-value $\leq 0.05$ was considered statistically significant. D After 45 weeks, spleens were also collected from each animal and weighed. Data are represented as the mean \pm standard deviation. $p$-values were calculated by Sidak's test and one-way ANOVA. A $p$-value $\leq 0.05$ was considered statistically significant. $\mathbf{E}$, F Liver tissues were processed for hematoxylin and eosin staining or immunohistochemical analysis for $\mathrm{Ki} 67$ and AFP protein expression levels. $\mathrm{N}$ and $\mathrm{T}$ depict normal and tumor regions, respectively $(\mathbf{E})$. More significant granuloma-like lesions were observed in the tumor tissues of DEN-treated WT mice compared with DEN/TSAHCtreated WT mice $(\mathbf{F})$. Data shown represent three independent experiments

and from those in DEN-treated KO mice $(p>0.9999$ and $p=0.2779$, respectively) (Fig. 4E). Meanwhile, only the perforin levels in WT mice were significantly decreased by DEN treatment $(p<0.0001)$, which were recovered by additional TSAHC treatment $(p=0.0453)$, suggesting NK cell activity appeared to be associated with TM4SF5 function/ activity-dependent carcinogenesis (Fig. 4E). That is, perforin expression from intrahepatic NK cells appeared to correlate with TM4SF5 function and activity during carcinogenesis, since TM4SF5 inhibition using TSAHC could lead to reduction of NK cells number or function for NK cell exhaustionlike phenotypes.

\section{TM4SF5 in hepatocytes modulated molecules stimulatory for NK cell surveillance}

We next examined how the NK cell cytotoxicity could depend on the TM4SF5 expression and/or activity in hepatocytes. NK92 (effector) cells were co-cultured with hepatocytes (target cancer cells) at different ratios before measurement of lysis of target cells. Endogenous TM4SF5 in Huh7 cells were suppressed (Fig. 5A), before the co-cultures.
Cytotoxicity of NK92 cells following the co-cultures increased when they targeted TM4SF5-suppressed cells, compared to non-suppressed target cells (Fig. 5B). Furthermore, we examined membrane proteins (or ligands) on the target hepatocytes, which could be involved in the regulation of NK cell cytotoxicity. qRT-PCR data from three different target cells, including Huh7, Hep3B, and HepG2, showed increased mRNA levels of co-stimulatory ligands on the target hepatocytes with suppression of endogenous TM4SF5, compared with those of non-suppressed (NS) target hepatocytes (Fig. 5C, red-highlighted). They include SLAMF6 [signaling lymphocytic activation molecule (SLAM) family member 6], SLAMF7 (SLAM family member 7), MICA, (major histocompatibility complex (MHC) I-related chain A), $M I C B$ (MHC class I related protein B), CADM1 (Cell adhesion molecule 1), ULBPI/2 (UL16-binding protein), and others.

Next, we investigated the TM4SF5-mediated effects on ligand levels and NK cell cytotoxicity after the co-culturing of NK92 effector cells with TM4SF5-expressing or -suppressed target hepatocytes. Analysis of cognate receptor expression of the NK92 cells after co-culture with TM4SF5suppressed target cells showed increased mRNA levels of natural killer receptor group 2 member D (NKG2D, stimulatory receptor for MICA/B and ULBP1/3 ligands), SLAMF6, and SLAMF7 (Fig. 5D). Consistently, suppression of TM4SF5 in the target cells resulted in increased NK92 cell cytotoxicity via elevated mRNA levels of PRF1, CD107a, TNF-a, FasL, and TRAIL in the NK92 cells (Fig. 5E). Coculturing of NK92 cells with TM4SF5-suppressed target HepG2 hepatocytes (another target cell line) also resulted in increased cytotoxicity of the NK92 cells (Fig. 5F). Following co-culture with TM4SF5-supppressed target cells, NK92 cells showed higher ERK1/2 activity (Fig. 5G), which is known to be associated with the upregulation of NK cell stimulatory receptors [32]. Consistently, when the target hepatocytes were treated with the TM4SF5 inhibitor, TSAHC, the NK92 cell cytotoxicity increased in a dosedependent manner (Fig. 5H). Additionally, we found that TM4SF5 expression levels in the target cancer cells showed an inverse relationship with the stimulatory ligand levels for NK cell cytotoxicity (Fig. 5I, left and middle). The levels of stimulatory ligands in TM4SF5-positive hepatocytes were increased or highly maintained after TSAHC treatment, whereas the levels appeared to decrease in TM4SF5-negative cells with TSAHC treatment (Fig. 5I, right). 
A Splenic immune cells

WT+Veh

$\mathrm{KO}+\mathrm{Veh}$

$W T+D E N+V e h$

$\mathrm{KO}+\mathrm{DEN}+\mathrm{Veh}$

$(n=6)$

$(n=6)$

$W T+D E N+T S A H C(n=4)$
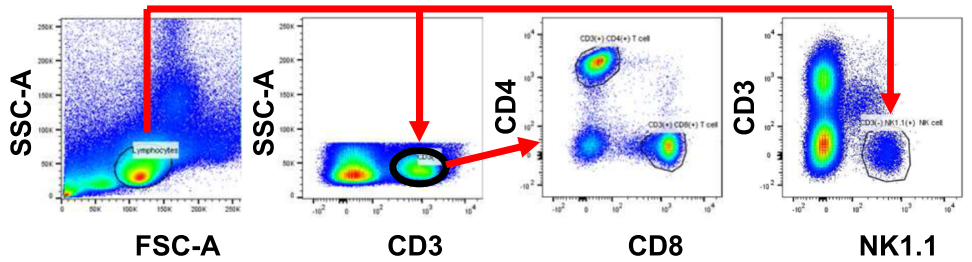

B
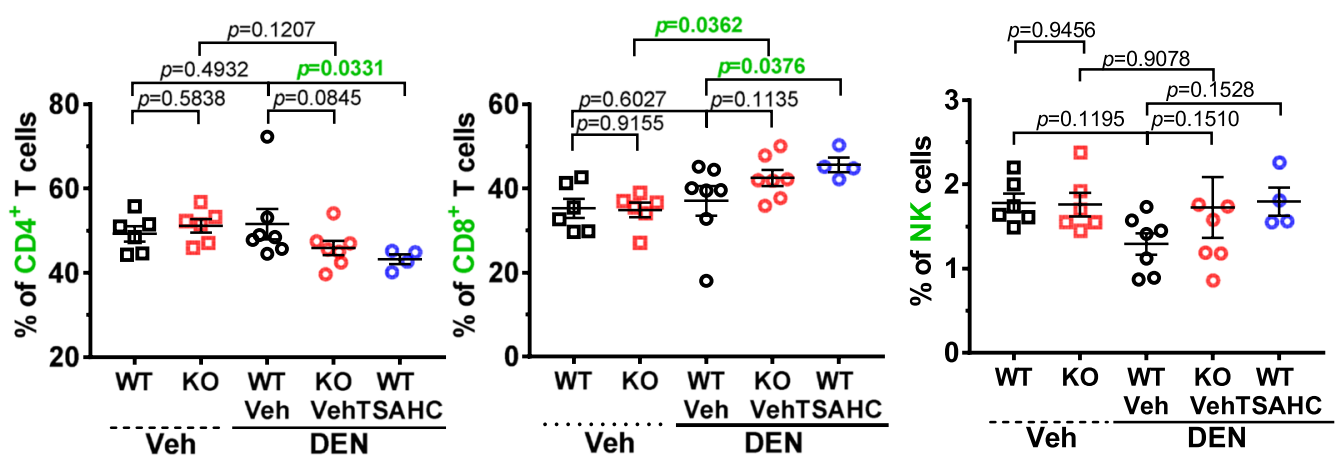

C

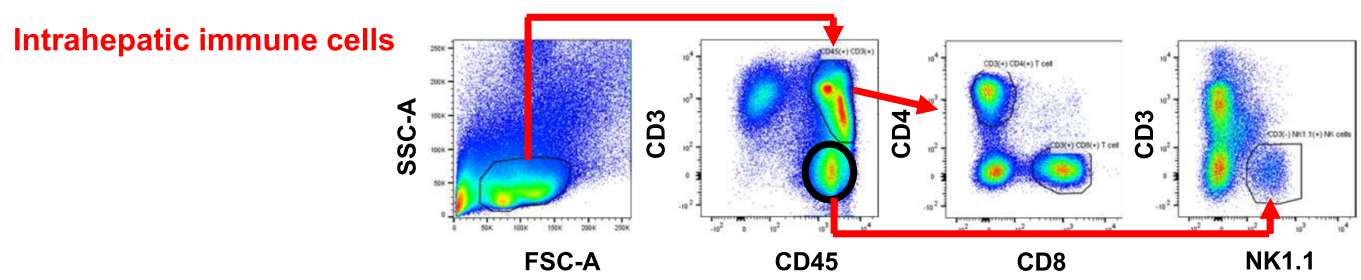

D
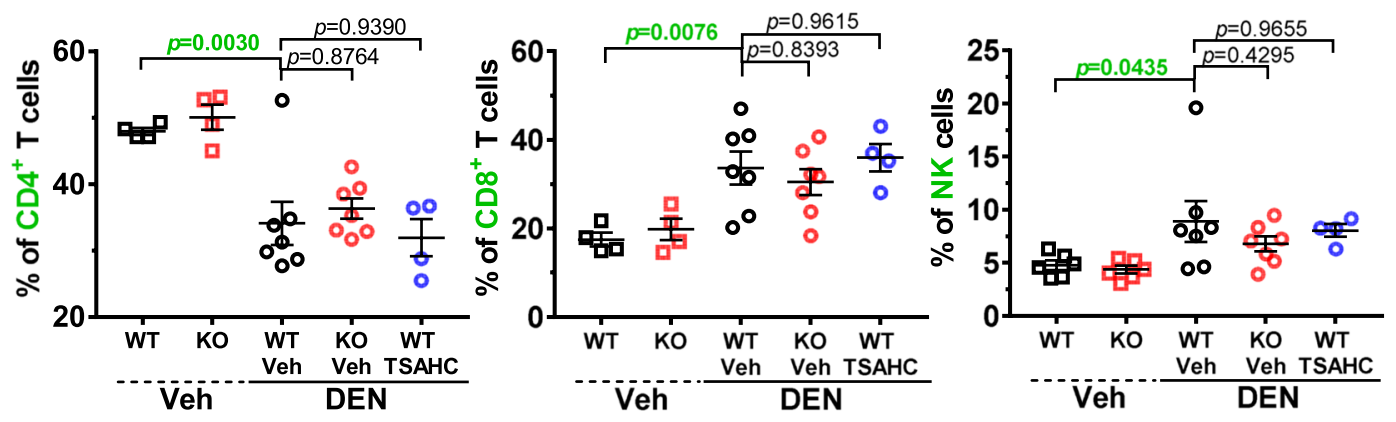

E
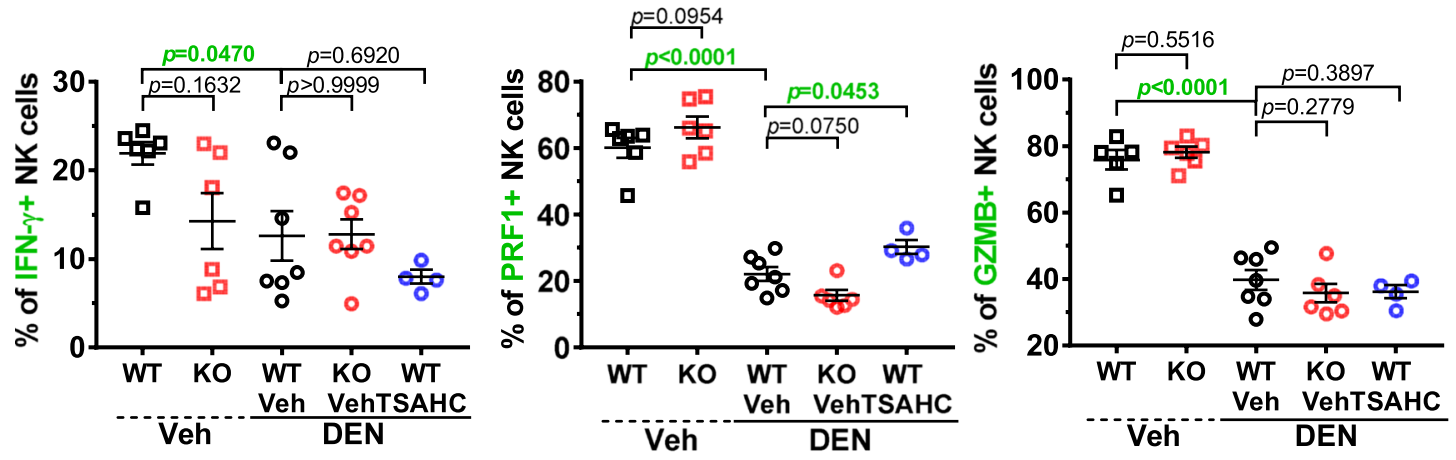
४Fig. 4 Natural killer cell involvement in diethylnitrosamine-induced, TM4SF5-dependent hepatic carcinogenesis. Spleen and liver tissues from WT and Tm $4 s f 5^{-1-}$ animals with or without diethylnitrosamine (DEN) treatment and in the absence or presence of 4'-( $p$-toluenesulfonylamido)-4-hydroxychalcone (TSAHC) co-treatment (described in Fig. 3A) were processed for splenic and hepatic immune cell population analysis by flow cytometry. A, B One outlier was excluded from the DEN/TSAHC-treated WT group. Splenic tissues for each experimental group were processed for immune cell population analysis using $\mathrm{CD}^{+} / \mathrm{CD}^{+}\left[\mathrm{T}\right.$-helper $(\mathrm{Th})$ cells], $\mathrm{CD}^{+} /$ $\mathrm{CD}^{+}\left(\mathrm{T}\right.$ cells), and $\mathrm{CD}^{3-} / \mathrm{NK} 1.1^{+}$[natural killer $(\mathrm{NK})$ cells] cell markers (A). The cell populations were graphed, and $p$ values were calculated by Sidak's test and one-way ANOVA. A $p$-value $\leq 0.05$ was considered statistically significant (B). C, D One outlier was excluded from the DEN/TSAHC-treated WT group. Hepatic tissues for each experimental group were processed for immune cell population analysis using $\mathrm{CD}^{+} / \mathrm{CD}^{+}, \mathrm{CD}^{+} / \mathrm{CD}^{+}$, and $\mathrm{CD}^{-} / \mathrm{CD}^{2} 5^{+} /$ $\mathrm{NK} 1.1^{+}$cell markers $(\mathbf{C})$. The intrahepatic cell populations were graphed, and $p$ values were calculated by Dunnett's test and one-way ANOVA. A $p$-value $\leq 0.05$ was considered statistically significant (D). E NK cells were analyzed for cytokine or granule secretion via antibody staining and flow cytometry. $p$ values were calculated by Sidak's test and one-way ANOVA. A $p$-value $\leq 0.05$ was considered statistically significant. Data represent three independent experiments or measurements

\section{TM4SF5 inhibitor abolished TM4SF5/ STAT3-mediated precancerous and cancerous phenotypes}

Because we showed that hepatic fibrosis/cirrhosis and cancer involves TM4SF5 (Fig. 2B, C), blockade of TM4SF5 function/activity may be a beneficial strategy for the treatment of TM4SF5-dependent liver diseases. Anti-TM4SF5 compound, TSAHC, was used to block DEN-induced carcinogenesis either at the beginning (DEN + TSAHC1) or during DEN treatment (DEN + TSAHC2). DEN treatment caused cancerous liver nodules, which were abolished by TSAHC treatment (Fig. 6A). Particularly, earlier TSAHC treatment (DEN + TSAHC1) led to increased survival and reduced $\mathrm{pY}^{705}$ STAT3 and laminin expression levels, whereas later TSAHC treatment (DEN + TSAHC2) did not reduce laminin expression, indicating that earlier TM4SF5 inhibition might be more effective to block the carcinogenesis (Fig. 6B, C). Consistent with these results, decreased laminin expression was evident in mice with earlier TSAHC treatment, but not in those with later TSAHC treatment, leading to a greater survival rate (Fig. 6B). These observations suggest that laminins play an important role in DEN-mediated hepatic carcinogenesis (Fig. 6C, D). Furthermore, public GEO data (GSE14520) analysis showed that TM4SF5 expression was highly elevated in HCC patients $(p<0.0001)$, and among samples those showing increased TM4SF5 expression in HCC, SLAMF7 ( $p<0.0001)$, MICA $(p=0.0161), U L B P 1$ $(p<0.0001)$, and $U L B P 2(p=0.0926)$ were oppositely decreased (Fig. 6E). These observations thus suggest that increased TM4SF5 expression in HCC samples over control counterparts could be linked to decreased expressions of stimulatory NK cell ligand/receptors. Taken together, these results suggest that blockade of TM4SF5 function/activity inhibits liver carcinogenesis, possibly by abolishing TM4SF5-mediated STAT3 activity, the expression of ECM factors, and improvement of NK cell cytotoxicity.

\section{Discussion}

This study provides evidence that TM4SF5-mediated STAT3 signaling promoted collagen I and laminin expression, which were involved in hepatic fibrosis/cirrhosis and eventual carcinogenesis in TM4SF5-transgenic and chemically induced mouse models. Furthermore, inhibition of TM4SF5 expression or activity and thereby of STAT3 activity abolished ECM production and cancerous phenotypes in the mice livers. DEN-mediated hepatic carcinogenesis was abolished by $T m 4 s f 5$ gene knockout or TM4SF5 protein inhibition, resulting in an increased population and thereby cytotoxic activity of NK cells. Meanwhile, during blockade of tumorigenesis via the TM4SF5 inhibition but not via TM4SF5-deficiency, $\mathrm{Th}$ and/or T cells might play roles in immune surveillance. Suppression or inhibition of TM4SF5 in target hepatocytes (cancer cells) increased the mRNA levels of stimulatory ligands in hepatocytes and cognate receptors in NK cells and also led to enhanced NK cell surveillance (Fig. 7). Therefore, TM4SF5 may be targeted using an anti-TM4SF5 reagent, such as a small compound TSAHC [25], or its antibody [19] as a potential NK cell-related immunotherapy for $\mathrm{HCC}$, in addition to immunotherapies against PD-1, PD-L1, or CTLA-1 [12]. Indeed, we have reported by mutation study and molecular modeling that TSAHC binding to TM4SF5 occurs at a region of the long extracellular loop (W124 to E129 of LEL) caused induced fit changes, leading to inhibitions of protein-protein interaction, L-arginine binding, and its downstream signaling activity [26]. TSAHC is quite specific for TM4SF5 at $0.3-5 \mu \mathrm{M}$, since TM4SF5-negative hepatocytes were not responsive to its treatment [25, 33, 34].

On the cell surface, there are certain domains in which specific membrane proteins and receptors are enriched to transduce specific intracellular signaling pathways. Such domains include focal adhesions, lipid rafts/caveolae, and tetraspanin-enriched microdomains (TERMs). Similar to focal adhesions with integrins that are engaged with the ECM and lipid rafts/caveolae that are enriched with glycosylphosphatidylinositol-linked proteins, TERMs are enriched with tetraspanins for massive protein-protein complexes that include tetraspanins, integrins, and growth factor receptors [35]. TM4SF5, a member of the transmembrane $4 \mathrm{~L}$ six family or L6 four transmembrane superfamily also forms TM4SF5-enriched microdomains $\left(\mathrm{T}_{5} \mathrm{ERMs}\right)$ [15]. Thus, TM4SF5 at $\mathrm{T}_{5}$ ERMs may function differentially 
A

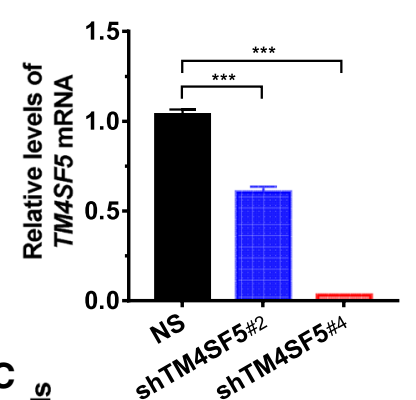

C
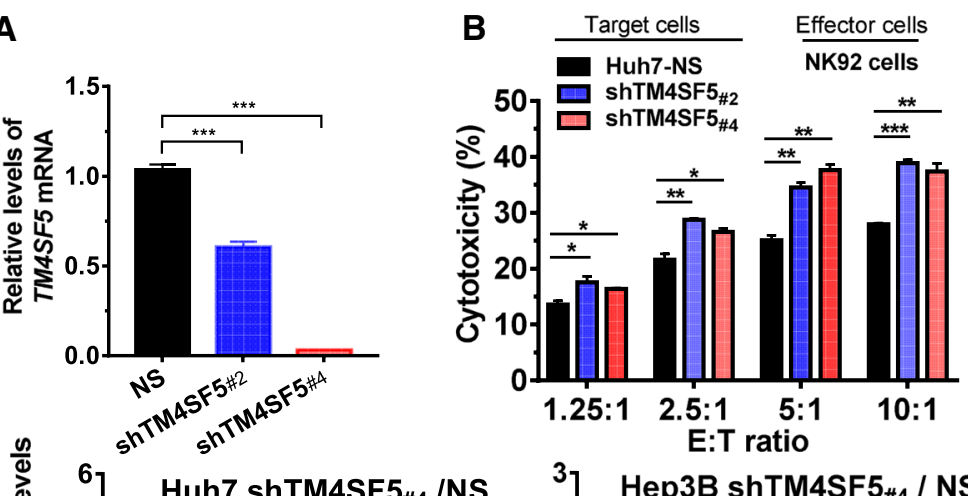
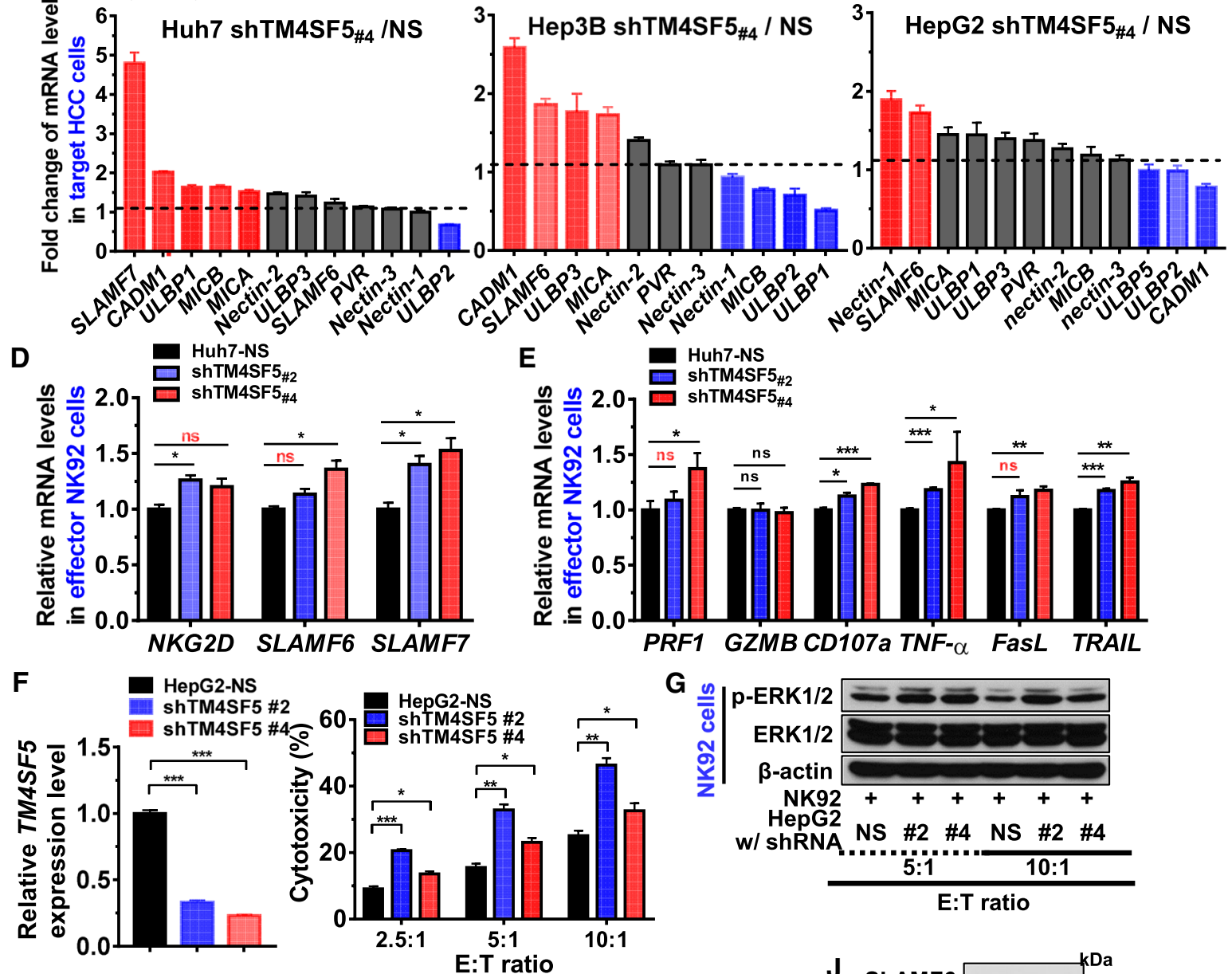

G

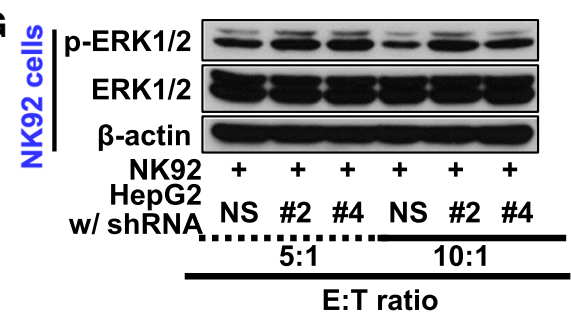

H
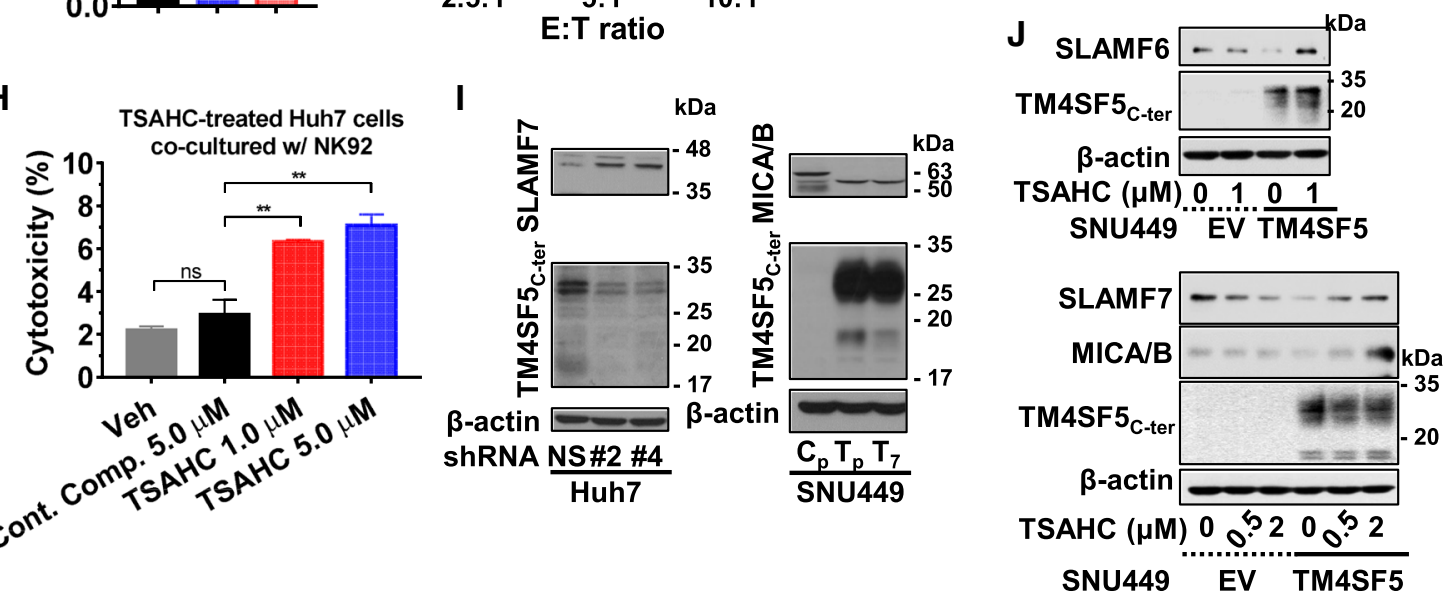
4Fig. 5 Immune evasion from natural killer cell cytotoxicity via TM4SF5 expression in hepatocytes. A-C Huh7 (A, B), Hep3B, and HepG2 cells with endogenous TM4SF5 expression (C) were infected with lentivirus for non-specific sequence (NS) or shTM4SF5 (targeting sequences \#2 and \#4 of TM4SF5) before expression level analysis by qRT-PCR for TM4SF5 or ligands for natural killer cell (NK) cell stimulation $(\mathbf{A}, \mathbf{C})$ or before a NK cell cytotoxicity assay using a co-culture system [at different ratios (E:T) of target (T) Huh7 hepatocytes and effector (E) NK92 cells] for $4 \mathrm{~h}(\mathbf{B})$. D-G Huh7 (D, E) or HepG2 (F, G) target (T) cells with or without TM4SF5 suppression $(\mathbf{A}, \mathbf{F}$, left) were co-cultured with NK92 effector $(\mathbf{E})$ cells for $4 \mathrm{~h}$ at an E:T ratio of 10:1 (D, E) or the indicated ratios $(\mathbf{F}, \mathbf{G})$ for NK92 cytotoxicity analysis (F, right), qRT-PCR (D-F, left), or whole-cell extract preparation prior to immunoblot analysis (G). H For NK92 cytotoxicity analysis, Huh7 target cells and NK92 effector cells were co-cultured for $6 \mathrm{~h}(\mathrm{E}: \mathrm{T}=10: 1)$, and 4'-( $p$-toluenesulfonylamido)4-hydroxychalcone (TSAHC) was administered at the indicated concentrations. Statistical significance is indicated by $*, * *, * * *$, or $* * * *$, which represent a $p$-value $\leq 0.05,0.01,0.001$, or 0.0001 , respectively, and ns indicates no significance, but red-highlighted ns indicates $0.05 \leq p$-values $\leq 0.10$. $p$-values were calculated by one-way analysis of variance (ANOVA) or one-tailed and unpaired Mann-Whitney $U$ tests. I, J Whole-cell extracts from hepatocytes with different TM4SF5 expression levels (I) and from hepatocytes transiently transfected with empty vector (EV) or TM4SF5 plasmids with or without TSAHC treatment at the indicated concentrations for $24 \mathrm{~h}(\mathbf{J})$ were prepared for immunoblot analysis of the indicated molecules. MICA/B blot from stable control SNU449C $\mathrm{C}_{\mathrm{p}}$ cells showed multiple

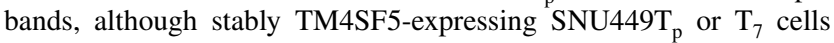
and other transiently transfected SNU449 cell lines showed a single band. Data shown represent three independent experiments

depending on cellular needs, via changes in binding partners or signaling activities/pathways to transduce diverse intracellular signaling pathways in a spatio-temporal manner [36]. TM4SF5 expression in hepatocytes promotes phosphorylations of FAK [37], c-SRC [20], STAT3 [21, 38], and mTOR/S6K1 [26]. TM4SF5 also binds EGFR [39], integrin $\alpha 5$ [38, 39], CD133 [34], CD151 [40], CD44 [41], and mTOR [26] membrane receptors or proteins. Interaction of TM4SF5 with integrin $\alpha 5$ induces VEGF secretion for hepatic angiogenesis via enhanced proliferation and migration of endothelial cells [38]. In addition, we have observed that TM4SF5 is involved in bidirectional crosstalks between hepatocytes and macrophages during development of NASH and is induced in differentiation/activation of macrophages or T cells (data not shown).

TM4SF5-mediated protein-protein complex formation at the $\mathrm{T}_{5}$ ERMs may serve as a signaling hub of diverse signaling pathways for gene expression or suppression at the transcriptional level. It may also modulate the expression levels of its binding partners via subcellular trafficking or compartmentalization prior to degradation or stabilization. In lung epithelial cells, TM4SF5 expression leads to suppression of ZEB2, which in turn increases alternative splicing factors for CD44 isoforms (from the standard CD44 form to CD44 variant form) during idiopathic pulmonary fibrosis [42]. Furthermore, TM4SF5 expression in hepatocytes leads to the downregulation of E-cadherin mRNA levels, thus promoting the loss of contact inhibition via epithelial-mesenchymal transition [17]. Here, we have shown that TM4SF5 decreased the mRNA levels of NK cell stimulatory receptors, such as $N K G 2 D$ (receptor of MICA/B and ULBP1/3 ligands), SLAMF6, and SLAMF7, and of hepatocyte cognate ligands, via immunoblot experiments and public GEO data analyses. Therefore, it can be likely that TM4SF5 transcriptionally regulated gene expression and was involved in tumor progression. Here, we also found that ERK1/2 phosphorylation increased in NK cells after co-culturing with TM4SF5suppressed target hepatocytes (Fig. 5G). Consistently, NK cell activation via the expression/activation of stimulatory receptors including those of the self-specific SLAM family for lymphocyte activation [43] and via lytic granule polarization and secretion [44], requires ERKs activation [32].

In addition, TM4SF5-mediated STAT3 activation appeared involved in excessive ECM production and regulation of stimulatory ligands and receptors levels for NK cell cytotoxicity. It is well known that collagen I expression is important for liver fibrosis [45]. Meanwhile, our present study shows that active STAT3 correlated with laminin and laminin $\gamma 2$ expression, in addition to collagen I, and also with subsequent hepatic carcinogenesis. Active STAT3 in hepatic inflammatory environment has been shown to induce ECM expressions by binding to their promoter regions [46]. Considerable immunostaining for laminin $\gamma 2$ is shown in the cytoplasm of hepatic carcinoma cells [47] and laminin $\gamma 2$ is suggested as a novel serum biomarker for HCC [48]. Indeed, TM4SF5 has been previously reported to be related to liver cancer progression, together with cell markers

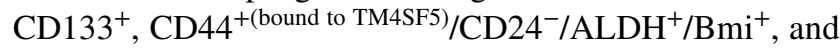
protein tyrosine phosphatase receptor type $\mathrm{F}\left(\mathrm{PTPRF}^{-}\right)$[34, $41]$, in addition to laminin $\gamma 2^{+}, \mathrm{AFP}^{+}, \mathrm{MCP}_{-} 1^{+}, \mathrm{CD}^{+} 4^{+}$, and $\mathrm{FUCA}^{+}$(AFU) shown in this study. TM4SF5 interacts with IL6R, leading to STAT3 activation independent of IL6 in hepatic cancer cells [21]. Because c-SRC is upstream of STAT3 [49] and TM4SF5 directly binds and activates c-SRC [20], TM4SF5 may therefore activate STAT3 in a ligandindependent manner. Indeed, IL6 activates STAT3 during HCC growth [50]. However, small in-frame deletions around the gp130 binding site for IL6 [51] and disruption of negative regulators of STAT3 such as SOCS3 and SHP1/2 [52, 53] are involved in ligand-independent STAT3 activations. Recently we reported that TM4SF5 activates STAT3 for the pathological progression to NASH-associated with fibrosis via hepatic inflammation-concomitant SOCS1/3 downregulation [24]. Here, a negative correlation was shown between TM4SF5 and SOCS1 expression in HCC patient groups. Therefore, TM4SF5 at $\mathrm{T}_{5}$ ERMs can promote STAT3 activity during the progression toward fibrotic and cancerous liver phenotypes. The blockade of TM4SF5-dependent 


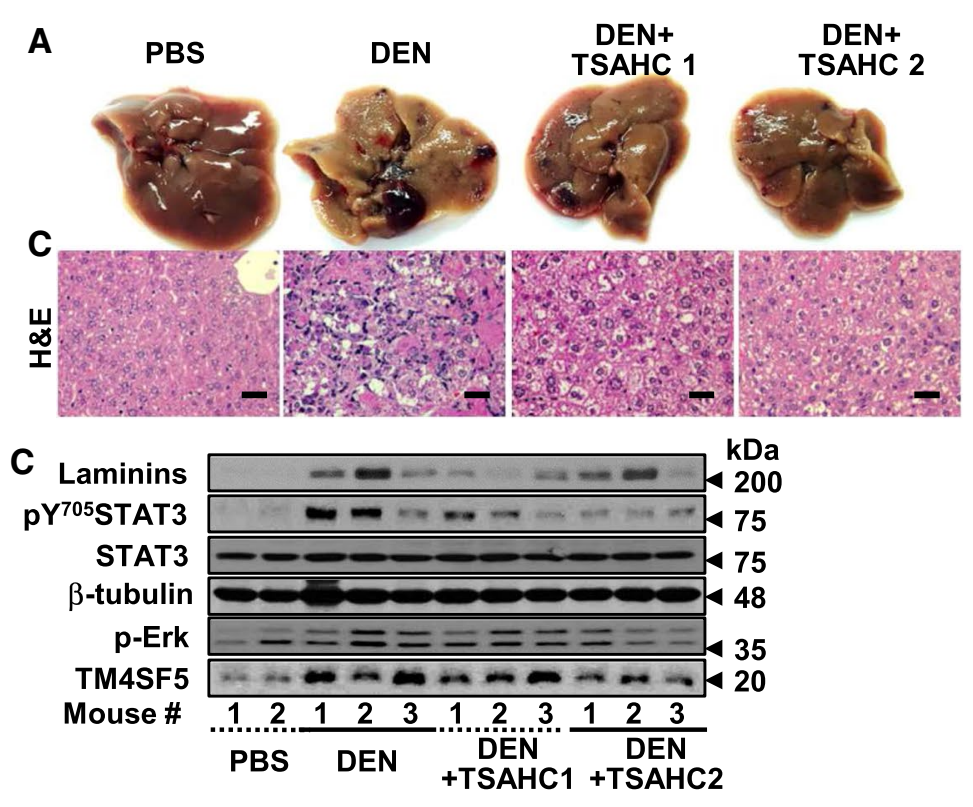

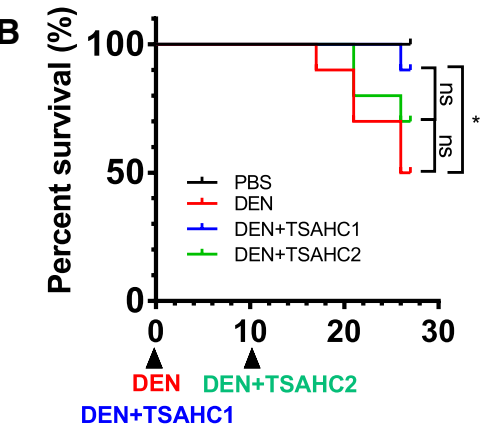

Time (weeks)

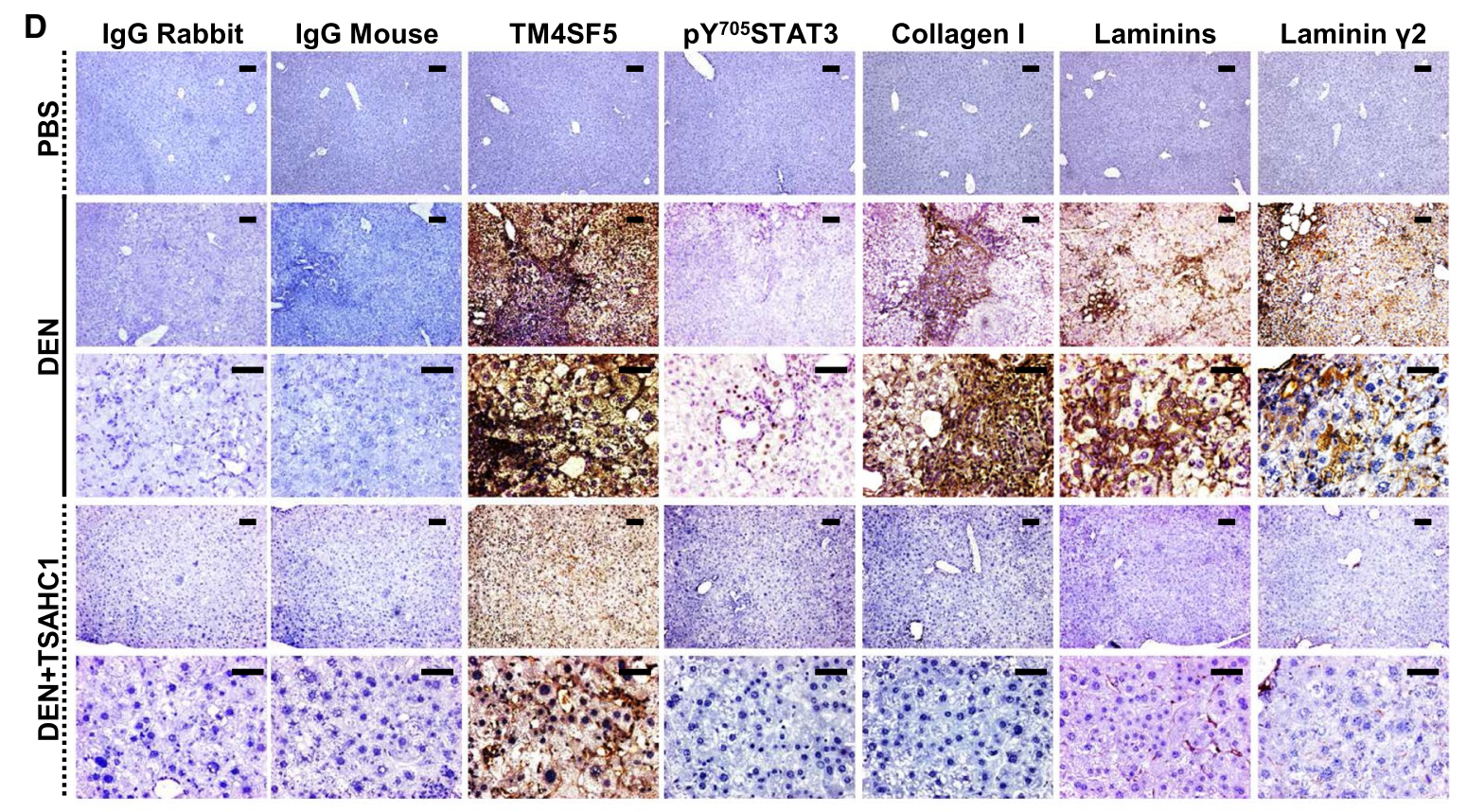

E

Roessler Liver2 (GSE14520)
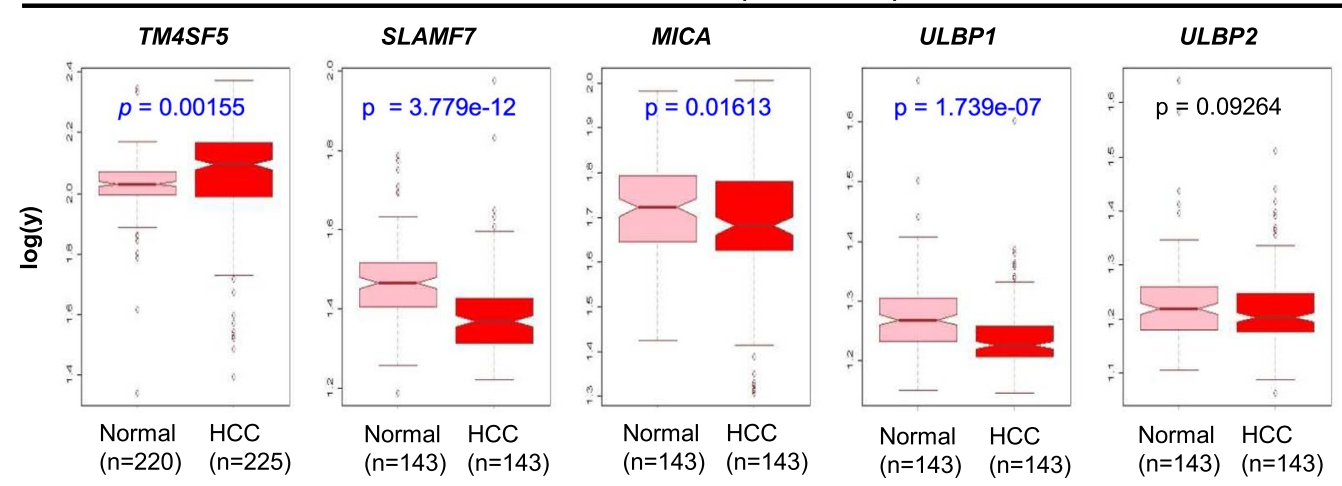
४Fig. 6 Abolishment of diethylnitrosamine-mediated precancerous and cancerous phenotypes by TM4SF5 inhibition. A-D Fiveweek-old BALB/c mice $(n \geq 5)$ were treated with diethylnitrosamine (DEN) for 27 weeks with or without intraperitoneal (IP) injection of DMSO as the vehicle control or $4^{\prime}$-( $p$-toluenesulfonylamido)4-hydroxychalcone (TSAHC), as explained in the Materials and Methods section. TSAHC was administered either at the beginning of DEN treatment (DEN + TSAHC1) or 10 weeks after DEN treatment (DEN + TSAHC2). Liver tissues were imaged and processed for hematoxylin and eosin staining (A). Survival rates of the mice in each experimental group were graphed $(\mathbf{B})$. Whole-tissue extracts were also prepared and processed for immunoblot analysis (C), or tissues were processed for immunohistochemical analysis of the indicated molecules (D). Immunoglobulins (IgG) from rabbit or mouse were used for the negative control stains without the primary antibodies. $p$-values were calculated by Gehan-Breslow-Wilcoxon test for ${ }^{*} p=0.0435$. $p$-value $>0.05$ was considered statistically insignificant (ns; $p=0.3679$ ). Data shown represent three independent experiments. E Gene expression profiles of liver cirrhosis [normal (Nor, $n=220)$ vs. HCC $(n=225)]$ from GSE14520 were analyzed and showed HCC with significantly increased TM4SF5 $(p<0.0001)$, and among the samples showing the increased TM4SF5 expressing in HCC $(n=143)$ SLAMF7 $(p<0.0001)$, MICA $(p=0.0161)$, and ULBP1 $(p<0.0001)$ were significantly decreased in HCC, compared with normal counterparts. Meanwhile, $U L B P 2$ was insignificantly decreased $(p=0.0926)$. $p$-values were calculated by the unpaired, two-tailed Student's $t$ tests

STAT3 activity and liver carcinogenesis by TSAHC treatment appeared further to be correlated with improved NK cell cytotoxicity.

The inhibition of TM4SF5 in (target) hepatocytes appeared to activate NK cell surveillance via different signaling pathways; (1) inactivation of STAT3 activity leading to apoptosis directly and activation/induction of stimulatory ligands in hepatocytes for NK cell surveillance indirectly, and (2) activation of ERKs in NK cells for increased SLAM and NKG2D receptor families for improvement of NK cell cytotoxicity. Consistently, STAT3-blocked (or inhibited) hepatocellular carcinoma cells showed upregulated NKG2D ligands (i.e., MICA/B and ULBPs), and thereby cytotoxicity of NK cells treated with supernatant from STAT3-blocked hepatocytes was augmented with a concomitant elevation of molecules associated with NK cytolysis [54, 55]. Interestingly, we have not found any cytokines, chemokines, growth factors, or extracellular matrix that could induce TM4SF5 in NK92 cells upon their treatment alone or in combinations (i.e., tested with approximately $40-50$ different factors, data not shown). Indeed, here TM4SF5 knock-out and inhibition (by TSAHC) led to less significant tumor formations in xenografts. However, NK cell cytotoxicity appeared functional upon TM4SF5 inhibition in vitro cells and in vivo animals but not upon Tm4sf5 gene knock-out. Although Th and T cells were shown to be involved in the TM4SF5 inhibitionmediated blockade of tumorigenesis, those immune cells appeared not to be critical for the DEN-mediated tumorigenesis in WT mice, compared to significantly less tumorigenesis in $\mathrm{KO}$ mice. Thus, we may speculate that in vivo complicated system without $T m 4 s f 5$ gene and related pathway may adopt other immune system (such as Th and/or $\mathrm{T}$ cells) or cytotoxic pathway (no survival/growth due to no TM4SF5-STAT3 activation). Presumably, conditional $T m 4 s f 5 \mathrm{KO}$ may be informative on whether the compensatory mechanisms may be the development of the immune system without TM4SF5 involvement. By the way, TM4SF5 in hepatocytes can still be targeted by an anti-TM4SF5 reagent, including TSAHC [25] and its antibody [19].

Altogether, we provide evidence that TM4SF5 plays an important role in the pathological progression of liver carcinogenesis via TM4SF5-mediated STAT3 signaling for ECM production. Concomitantly, TM4SF5 mediates the downregulation of stimulatory membrane ligands on target hepatocytes that stimulate NK cell cytotoxicity, leading to NK cell immune exhaustion-like phenotypes including reduction of NK cells number or function upon TSAHC treatment. Thus, TM4SF5 (as a molecule to suppress NK surveillance) is a promising target for the treatment of advanced liver diseases. 


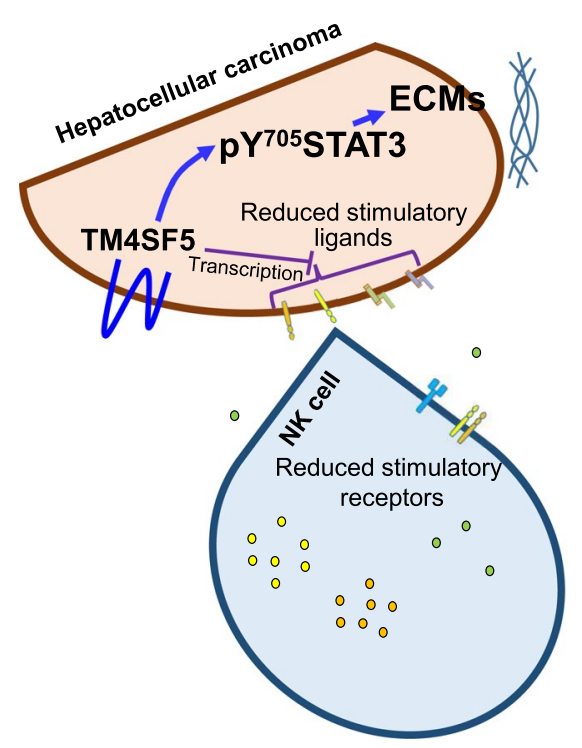

Inhibited NK cell cytotoxicity

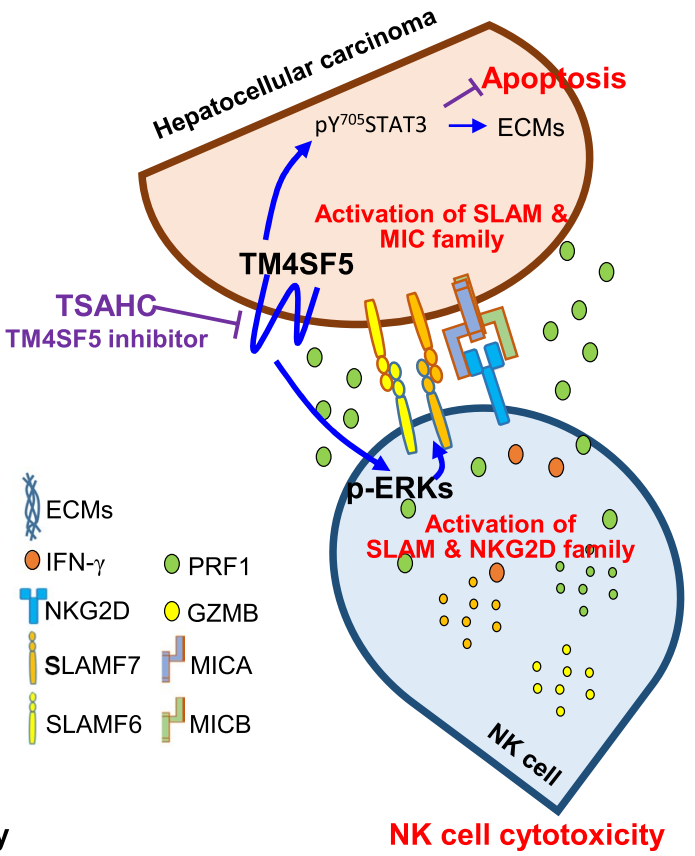

Fig. 7 Working models for TM4SF5-mediated suppression of NK cell surveillance. (Left) Highly expressed TM4SF5 in liver cancer cells triggers intracellular signaling for the downregulation of surface ligand-related factors at the transcriptional level, which leads to inhibited NK cell cytotoxicity [i.e., immune evasion from natural killer (NK) cells], in addition to enhanced TM4SF5-pY ${ }^{705} \mathrm{~S}$ TAT3 signaling and extracellular matrix production for the promotion of precancerous (fibrotic/cirrhotic) and cancerous phenotypes. (Right) Inhibition of TM4SF5 using anti-TM4SF5 compound, 4'-( $p$-toluenesulfonylamido)-4-hydroxychalcone (TSAHC), upregulates the immune checkpoints between NK cells and hepatocytes,

Author contributions HS, JHR, and EMK performed most experiments; HJL helped animal experiments; JWJ, JEK, HSL, EAS, and YP helped with imaging experiments and with reagents; JHL and JHY helped with clinical tissue samples; SK helped with analysis on NK cell cytotoxicity and discussion on manuscript; JWL designed the experiments and wrote the manuscript.

Funding This work was supported by Basic Science Research Program through the National Research Foundation of Korea (NRF) funded by the Ministry of Science, ICT and Future Planning (NRF2020R1I1A1A01070020 to EMK, NRF-2018M3A9C8020027 to SK and JWL, 2020R1A2C3008993, and NRF-2021M3A9D3024752 to JWL) for the Tumor Microenvironment GCRC (2011-0030001) to JWL

Data availability Upon written requests to the corresponding author, the data and materials can be available.

Code availability Not applicable.

\section{Declarations}

Conflict of interest The authors declare no potential conflicts of interest. including SLAM, NKG2D, and MICA/B member receptors and ligands, which leads to the stimulation of NK cell cytotoxic receptors and enhanced NK cell cytotoxicity. Activation of NK cell cytotoxicity upon TM4SF5 inhibition by TSAHC treatment appeared to cause (1) inactive STAT3-mediated activation of ligands in hepatocytes stimulatory for NK cell cytotoxicity [54] and (2) ERK activation-mediated increases in stimulatory receptors in NK cells neighbored with TM4SF5-inhibited hepatocytes (Fig. 5G). TM4SF5-mediated STAT3 activation and extracellular matrix production are blocked by TSAHC treatment, which leads to NK cell cytotoxic activity as a potential immunotherapy for TM4SF5-positive liver cancer

Ethics approval All animal procedures were performed in accordance with the Seoul National University Laboratory Animal Maintenance Manual and were approved by the IRB of the Institute of Laboratory Animal Resources Seoul National University (SNU-IACUC) (SNU140423-11-7, SNU-130911-2-3, SNU-170920-9, SNU-190122-6-3).

Consent to participate Not applicable.

Consent for publication All authors have consented for a publication in the CMLS.

Open Access This article is licensed under a Creative Commons Attribution 4.0 International License, which permits use, sharing, adaptation, distribution and reproduction in any medium or format, as long as you give appropriate credit to the original author(s) and the source, provide a link to the Creative Commons licence, and indicate if changes were made. The images or other third party material in this article are included in the article's Creative Commons licence, unless indicated otherwise in a credit line to the material. If material is not included in the article's Creative Commons licence and your intended use is not permitted by statutory regulation or exceeds the permitted use, you will need to obtain permission directly from the copyright holder. To view a copy of this licence, visit http://creativecommons.org/licenses/by/4.0/. 


\section{References}

1. Tu T, Calabro SR, Lee A et al (2015) Hepatocytes in liver injury: victim, bystander, or accomplice in progressive fibrosis? J Gastroenterol Hepatol 30:1696-1704

2. Bonnans C, Chou J, Werb Z (2014) Remodelling the extracellular matrix in development and disease. Nat Rev Mol Cell Biol 15:786-801

3. Tovoli F, Casadei-Gardini A, Benevento F, Piscaglia F (2019) Immunotherapy for hepatocellular carcinoma: a review of potential new drugs based on ongoing clinical studies as of 2019. Dig Liver Dis 51:1067-1073

4. Lu L, Jiang J, Zhan M et al (2021) Targeting tumor-associated antigens in hepatocellular carcinoma for immunotherapy: past pitfalls and future strategies. Hepatology 73:821-832

5. Wang Z, Aguilar EG, Luna JI et al (2019) Paradoxical effects of obesity on $\mathrm{T}$ cell function during tumor progression and PD-1 checkpoint blockade. Nat Med 25:141-151

6. Qu J, Mei Q, Liu L et al (2021) The progress and challenge of anti-PD-1/PD-L1 immunotherapy in treating non-small cell lung cancer. Ther Adv Med Oncol 13:1758835921992968

7. Cooper MA, Fehniger TA, Turner SC et al (2001) Human natural killer cells: a unique innate immunoregulatory role for the CD56 (bright) subset. Blood 97:3146-3151

8. Voskoboinik I, Whisstock JC, Trapani JA (2015) Perforin and granzymes: function, dysfunction and human pathology. Nat Rev Immunol 15:388-400

9. Wallin RP, Screpanti V, Michaelsson J et al (2003) Regulation of perforin-independent NK cell-mediated cytotoxicity. Eur J Immunol 33:2727-2735

10. Morvan MG, Lanier LL (2016) NK cells and cancer: you can teach innate cells new tricks. Nat Rev Cancer 16:7-19

11. Wu SY, Fu T, Jiang YZ, Shao ZM (2020) Natural killer cells in cancer biology and therapy. Mol Cancer 19:120

12. Liu X, Qin S (2019) Immune checkpoint inhibitors in hepatocellular carcinoma: opportunities and challenges. Oncologist 24:S3-S10

13. Pineiro Fernandez J, Luddy KA, Harmon C, O'Farrelly C (2019) Hepatic tumor microenvironments and effects on NK cell phenotype and function. Int J Mol Sci 20:4131

14. Wright MD, Ni J, Rudy GB (2000) The L6 membrane proteins-a new four-transmembrane superfamily. Protein Sci 9:1594-1600

15. Lee JW (2015) Transmembrane 4 L six family member 5 (TM4SF5)-mediated epithelial-mesenchymal transition in liver diseases. Int Rev Cell Mol Biol 319:141-163

16. Kang M, Jeong SJ, Park SY et al (2012) Antagonistic regulation of transmembrane 4 L6 family member 5 attenuates fibrotic phenotypes in $\mathrm{CCl}_{4}$-treated mice. FEBS J 279:625-635

17. Lee SA, Lee SY, Cho IH et al (2008) Tetraspanin TM4SF5 mediates loss of contact inhibition through epithelial-mesenchymal transition in human hepatocarcinoma. J Clin Investig 118:1354-1366

18. Muller-Pillasch F, Wallrapp C, Lacher U et al (1998) Identification of a new tumour-associated antigen TM4SF5 and its expression in human cancer. Gene 208:25-30

19. Ahn H-M, Ryu J, Song JM et al (2017) Anti-cancer activity of novel TM4SF5-targeting antibodies through TM4SF5 neutralization and immune cell-mediated cytotoxicity. Theranostics 7:594-613

20. Jung O, Choi YJ, Kwak TK et al (2013) The COOH-terminus of TM4SF5 in hepatoma cell lines regulates c-Src to form invasive protrusions via EGFR Tyr845 phosphorylation. Biochim Biophys Acta 1833:629-642
21. Ryu J, Kang M, Lee M-S et al (2014) Cross talk between the TM4SF5/Focal Adhesion Kinase and the Interleukin-6/STAT3 pathways promotes immune escape of human liver cancer cells. Mol Cell Biol 34:2946-2960

22. Kang M, Choi S, Jeong SJ et al (2012) Cross-talk between TGF $\beta 1$ and EGFR signalling pathways induces TM4SF5 expression and epithelial-mesenchymal transition. Biochem $\mathbf{J}$ 443:691-700

23. Mahler JF, Stokes W, Mann PC et al (1996) Spontaneous lesions in aging FVB/N mice. Toxicol Pathol 24:710-716

24. Ryu J, Kim E, Kang M-K et al (2020) Differential TM4SF5-mediated SIRT1 modulation and metabolic signaling in nonalcoholic steatohepatitis progression. J Pathol 253:55-67

25. Lee SA, Ryu HW, Kim YM et al (2009) Blockade of four-transmembrane L6 family member 5 (TM4SF5)-mediated tumorigenicity in hepatocytes by a synthetic chalcone derivative. Hepatology 49:1316-1325

26. Jung JW, Macalino SJY, Cui M et al (2019) Transmembrane 4 L six family member 5 senses arginine for mTORC1 signaling. Cell Metab 29:1306-1319

27. Zhong W, Shen WF, Ning BF et al (2009) Inhibition of extracellular signal-regulated kinase 1 by adenovirus mediated small interfering RNA attenuates hepatic fibrosis in rats. Hepatology 50:1524-1536

28. Wang WW, Ang SF, Kumar R et al (2013) Identification of serum monocyte chemoattractant protein- 1 and prolactin as potential tumor markers in hepatocellular carcinoma. PLoS One 8:e68904

29. Giardina MG, Matarazzo M, Varriale A et al (1992) Serum $\alpha$-Lfucosidase. A useful marker in the diagnosis of hepatocellular carcinoma. Cancer 70:1044-1048

30. Dai L, Peng XX, Tan EM, Zhang JY (2016) Tumor-associated antigen CAPER $\alpha$ and microvessel density in hepatocellular carcinoma. Oncotarget 7:16985-16995

31. Zhao YJ, Ju Q, Li GC (2013) Tumor markers for hepatocellular carcinoma. Mol Clin Oncol 1:593-598

32. Meza Guzman LG, Keating N, Nicholson SE (2020) Natural killer cells: tumor surveillance and signaling. Cancers (Basel) 12:952

33. Lee SA, Lee MS, Ryu HW et al (2011) Differential inhibition of transmembrane $4 \mathrm{~L}$ six family member 5 (TM4SF5)-mediated tumorigenesis by TSAHC and sorafenib. Cancer Biol Ther $11: 330-336$

34. Kim S, Cho CY, Lee D et al (2018) CD133-induced TM4SF5 expression promotes sphere growth via recruitment and blocking of protein tyrosine phosphatase receptor type F (PTPRF). Cancer Lett 438:219-231

35. Lee JW (2014) TM4SF5-mediated protein-protein networks and tumorigenic roles. BMB Rep 47:483-487

36. Jung JW, Kim JE, Kim E, Lee JW (2020) Amino acid transporters as tetraspanin TM4SF5 binding partners. Exp Mol Med 52:7-14

37. Jung O, Choi S, Jang SB et al (2012) Tetraspan TM4SF5-dependent direct activation of FAK and metastatic potential of hepatocarcinoma cells. J Cell Sci 125:5960-5973

38. Choi S, Lee S-A, Kwak TK et al (2009) Cooperation between integrin $\alpha 5$ and tetraspan TM4SF5 regulates VEGF-mediated angiogenic activity. Blood 113:1845-1855

39. Kim HJ, Kwon S, Nam SH et al (2017) Dynamic and coordinated single-molecular interactions at TM4SF5-enriched microdomains guide invasive behaviors in 2- and 3-dimensional environments. FASEB J 31:1461-1481

40. Kang M, Ryu J, Lee D et al (2014) Correlations between Transmembrane 4 L6 family member 5 (TM4SF5), CD151, and CD63 in liver fibrotic phenotypes and hepatic migration and invasive capacities. PLoS One 9:e102817 
41. Lee D, Na J, Ryu J et al (2015) Interaction of tetraspan (in) TM4SF5 with CD44 promotes self-renewal and circulating capacities of hepatocarcinoma cells. Hepatology 61:1978-1997

42. Kim JE, Kim HJ, Jung JW et al (2019) TM4SF5-mediated CD44v8-10 splicing variant promotes survival of type II alveolar epithelial cells during idiopathic pulmonary fibrosis. Cell Death Dis 10:645

43. Chen X, Trivedi PP, Ge B et al (2007) Many NK cell receptors activate ERK2 and JNK1 to trigger microtubule organizing center and granule polarization and cytotoxicity. Proc Natl Acad Sci USA 104:6329-6334

44. Chen X, Allan DSJ, Krzewski K et al (2006) CD28-stimulated ERK2 phosphorylation is required for polarization of the microtubule organizing center and granules in YTS NK cells. Proc Natl Acad Sci USA 103:10346-10351

45. Yang MC, Wang CJ, Liao PC et al (2014) Hepatic stellate cells secretes type I collagen to trigger epithelial mesenchymal transition of hepatoma cells. Am J Cancer Res 4:751-763

46. Song DG, Kim D, Jung JW et al (2018) Glutamyl-prolyl-tRNA synthetase induces fibrotic extracellular matrix via both transcriptional and translational mechanisms. FASEB J 33:4341-4354

47. Aishima S, Matsuura S, Terashi T et al (2004) Aberrant expression of laminin $\gamma 2$ chain and its prognostic significance in intrahepatic cholangiocarcinoma according to growth morphology. Mod Pathol 17:938-945

48. Kiyokawa H, Yasuda H, Oikawa R et al (2017) Serum monomeric laminin- $\gamma 2$ as a novel biomarker for hepatocellular carcinoma. Cancer Sci 108:1432-1439
49. Jung JE, Lee HG, Cho IH et al (2005) STAT3 is a potential modulator of HIF-1-mediated VEGF expression in human renal carcinoma cells. FASEB J 19:1296-1298

50. Wang H, Lafdil F, Kong X, Gao B (2011) Signal transducer and activator of transcription 3 in liver diseases: a novel therapeutic target. Int J Biol Sci 7:536-550

51. Rebouissou S, Amessou M, Couchy G et al (2009) Frequent inframe somatic deletions activate gp130 in inflammatory hepatocellular tumours. Nature 457:200-204

52. Riehle KJ, Campbell JS, McMahan RS et al (2008) Regulation of liver regeneration and hepatocarcinogenesis by suppressor of cytokine signaling 3. J Exp Med 205:91-103

53. He G, Yu GY, Temkin V et al (2010) Hepatocyte IKK $\beta / \mathrm{NF}-\kappa \mathrm{B}$ inhibits tumor promotion and progression by preventing oxidative stress-driven STAT3 activation. Cancer Cell 17:286-297

54. Sun X, Sui Q, Zhang C et al (2013) Targeting blockage of STAT3 in hepatocellular carcinoma cells augments NK cell functions via reverse hepatocellular carcinoma-induced immune suppression. Mol Cancer Ther 12:2885-2896

55. Juengpanich S, Shi L, Iranmanesh Y et al (2019) The role of natural killer cells in hepatocellular carcinoma development and treatment: a narrative review. Transl Oncol 12:1092-1107

Publisher's Note Springer Nature remains neutral with regard to jurisdictional claims in published maps and institutional affiliations. 